\title{
Turbulent mixing at a shear-free density interface
}

\author{
By IMAD A. HANNOUN \\ Jaycor, P.O. Box 85154, San Diego, CA 92138-9259, USA
}

AND E. JOHN LIST

W. M. Keck Laboratory of Hydraulics \& Water Resources, California Institute of Technology, Pasadena, CA 91125, LTSA

(Received 31 October 1986 and in revised form 13 August 1987)

The interaction of a sharp density interface with oscillating-grid-induced shear-free turbulence was experimentally investigated. A linear photodiode array was used in conjunction with laser-induced fluorescence to measure the concentration of dye that was initially only in the less dense layer. A laser-Doppler velocimeter was used to measure the vertical velocity in and above the density interface at a point where the dye concentration was also measured. Potential refractive-index-fluctuation problems were avoided using solutes that provided a homogeneous optical environment across the density interface. Internal wave spectra, amplitudes and velocities, as well as the vertical mass flux were measured. The results indicate that mixing occurs in intermittent bursts and that the gradient (local) Richardson number remains constant for a certain range of the overall Richardson number $R_{j}$, defined in terms of an integral lengthscale, buoyancy jump and turbulence intensity. The spectra of the internal waves decay as $f^{-3}$ at frequencies below the maximum Brunt-Väisälä frequency. These findings give support to a model for oceanic mixing proposed by Phillips (1977) in which the internal waves are limited in their spectral density by sporadic local instabilities and breakdown to turbulence. The results also indicate that, for a certain $R_{j}$ range, the thickness of the interfacial layer (normalized by the integral lengthscale of the turbulence) is a decreasing function of $R_{j}$. At sufficiently high $R_{j}$ the interfacial thickness becomes limited by diffusive effects. Finally, we discuss a simple model for entrainment at a density interface in the presence of shearfree turbulence.

\section{Introduction and literature review}

Turbulent mixing, and especially turbulent mixing within density-stratified fluids, continues to be a perplexing problem in environmental and geophysical fluid dynamics. Of particular interest is the mixing in the neighbourhood of relatively sharp density interfaces in the oceans, lakes, reservoirs and the atmosphere. In environmental fluid mechanics, an understanding of these mixing processes is essential in determining water quality in water bodies containing a sharp density interface. The presence of such an interface, by suppressing fluid turbulence, limits the downward transfer of pollutants and gases such as carbon dioxide and oxygen from the upper mixed layer (Fischer et al. 1979), and the upward transport of nutrients to organisms that live in the surface layer (Scavia \& Robertson 1981). This has important consequences in the design of wastewater and thermal outfalls. In the atmosphere severe air-pollution problems may result from the presence of thermal inversions that inhibit the vertical transport of pollutants. Here again, a knowledge 
of the exchange processes across the inversion layer can be important in predicting and controlling air quality in the lower atmosphere.

Many prior attempts (Rouse \& Dodu 1955; Cromwell 1960; Bouvard \& Dumas 1967; Turner 1968; Kato \& Phillips 1969; Wu 1973; Linden 1973, 1975; Crapper \& Linden 1974; Thompson \& Turner 1975; Wolanski \& Brush 1975; Hopfinger \& Toly 1976; Kantha, Phillips \& Azad 1977; Gartrell 1979; McDougall 1979; Kantha \& Long 1980 ; Folse, Cox \& Schexnayder 1981 ; Hopfinger \& Linden 1982 ; Fernando \& Long 1983, 1985), both experimental and theoretical, have been made at observing and modelling the mixing across sharp density interfaces. The laboratory experiments tend to fall into three categories: (i) those where the mixed layer is driven over the non-turbulent layer thus creating a mean velocity shear, (ii) those where the turbulence is generated by a vertically oscillating grid and hence exhibit no mean shear, and (iii) those where the turbulence is generated by convective heating or cooling at a surface. Extensive discussion of the progress of research in these areas is given in the reviews by Turner (1979) and Phillips (1977).

\subsection{Oscillating-grid experiments}

For oscillating-grid experiments Turner (1979) proposed that the entrainment velocity $u_{\mathrm{e}}$, defined as $u_{\mathrm{e}}=\mathrm{d} D / \mathrm{d} t$, where $D$ is the depth of the upper layer, is given by a simple law of the form

$$
u_{\mathrm{e}} /\left(\sigma_{u}\right)_{\mathrm{o}} \sim R_{i}^{-n} \quad\left(R_{i}>7\right),
$$

where $\sigma_{u}$ is the r.m.s. horizontal velocity and the subscript o here and subsequently refers to the quantity being measured in a homogeneous fluid at the same distance from the oscillating grid as the density interface. $R_{i}$ is an overall Richardson number defined by $R_{i}=\Delta b l_{0} /\left(\sigma_{u}\right)_{0}^{2}$, where $\Delta b$ is the buoyancy jump and $l_{\mathrm{o}}$ is an integral lengthscale of the turbulence defined as the area under the auto-correlation coefficient curve for the horizontal velocity component. Turner (1979) suggested that $n=\frac{3}{2}$ when the stratification was due to salt. When the density stratification resulted from a temperature gradient, the value of $n$ was found to be about 1 . Turner (1979) suggested that viscosity differences cannot be used to explain the different values of $n$. He proposed that the large differences in $u_{\mathrm{e}}$ that occur when using salt and heat as the stratifying agents can only be explained by consideration of the molecular diffusion of mass and heat as defined by the appropriate diffusivity $\kappa$. He added a second dimensionless number, a Péclet number $P_{e}=\left(\sigma_{u}\right)_{0} l_{\mathrm{o}} / \kappa$, and proposed that the entrainment velocity would be a function of both an overall Richardson number and this Péclet number. Turner (1968) suggested that

$$
u_{\mathrm{e}} /\left(\sigma_{u}\right)_{\mathrm{o}}=R_{i}^{-1}\left(c+R_{i} P_{e}\right)^{-\frac{1}{2}},
$$

where $c$ is a constant. When $P_{e}$ is small this relationship reduces to $u_{\mathrm{e}} /\left(\sigma_{u}\right)_{0} \sim R_{i}^{-1}$. When $P_{e}$ is large (1.2) becomes $u_{\mathrm{e}} /\left(\sigma_{u}\right)_{0} \sim R_{i}^{-\frac{3}{2}} P_{e}^{-\frac{1}{2}}$.

After Turner's work clearly showed different entrainment rates for heat and salt interfaces, other investigators attempted to explain the differences both theoretically and experimentally. A problem that confronted experimentalists was that the molecular diffusivities of heat and salt differ by about two orders of magnitude and that other suitable solutes with intermediate diffusivities are unavailable. This made the systematic study of the problem over a continuous range of $P_{e}$ difficult. As a result, the available experimental data tends to be at either low $P_{e}$ (using heat) or rather high $P_{e}$ (using salt or other solutes with comparable diffusivities). The results for each of these ranges, as well as those obtained for very high $R_{i}$, will be discussed next. 


\subsubsection{High-Péclet-number investigations}

Several attempts have been made to explain the observed entrainment law near high $P_{e}$ density interfaces. Linden (1973) experimentally investigated the effects of a vortex ring impinging on a sharp density interface and developed a model for the ring-interface interaction. The model was then extended to calculate the rate of entrainment at a density interface in the presence of oscillating-grid turbulence. This was done with the conjecture that, in this particular case, a vortex ring appears to be a good approximation to a turbulent eddy. More recently, Linden (1975) proposed another model in which he postulated that the rate of change of potential energy due to mixing is proportional to the total kinetic energy flux available at the interface. This explanation leads to a $u_{\mathrm{e}} /\left(\sigma_{u}\right)_{\mathrm{o}} \sim R_{i}^{-1}$ relationship. As much as this simple idea is physically attractive, other investigations (Turner 1968; Hopfinger \& Toly 1976; Fernando \& Long 1983) found that the entrainment rate is much slower than predicted by Linden (1975). Long (1978) incorporated some of the ideas of the rapid distortion theory of Hunt \& Graham (1978) and suggested that the turbulent eddies tend to flatten as they approach the density interface, thus rendering the turbulence anisotropic. This is partly supported by a study of vortices impinging on a density interface (Linden 1973) and Dickey et al.'s (1984) work on turbulent eddies below an air-water interface. Long's model also postulates that the entrainment occurs owing to the breaking of interfacial waves in the interfacial layer. This wave-breaking is considered to occur through a resonance mechanism in which the forcing is accomplished by the 'quasi-isotropic' eddies of the mixed layer acting on the upper surface of the interfacial layer (Fernando \& Long 1983).

The thickness of the interfacial layer $h$ in salt-stratified experiments was determined by Crapper \& Linden (1974), who used both a travelling conductivity probe and shadowgraph techniques. Although $l_{0}$ was not measured directly, they found $h / l_{0}$ to be independent of $R_{i}$ and given by $h / l_{0} \sim 1-1.5$. Moreover, they observed that there was no significant difference between their time-averaged and instantaneous density profiles. Hopfinger \& Toly (1976) also measured $h / l_{\mathrm{o}}$ and found a clear dependence on $R_{i}$, which contradicts the observations of Crapper \& Linden. Hopfinger \& Toly also measured $h_{\mathrm{s}}$, the "static' thickness of the interface after the stirrer had been stopped and the turbulent motion had calmed down. They observed that $h_{\mathrm{s}} \sim l_{\mathrm{o}}$ and that the relative difference in the dynamic and static layer thickness $\left(h-h_{\mathrm{s}}\right) / h_{\mathrm{s}} \sim R_{i}^{-1}$. Measurements made by Fernando \& Long (1985) using a travelling conductivity probe suggest that at low $R_{i}$ an instantaneous density profile is not a good indicator of the average profile. They also find that $h / D$, where $D$ is the depth of the upper layer measured from a virtual origin, is constant but is considerably less than that obtained by Crapper \& Linden. By using shadowgraph techniques, Wolanski \& Brush (1975) also found $h / D$ to be a constant whose value is comparable to that in the experiments of Fernando \& Long (1985).

As evident from the above discussion, there is considerable confusion regarding the thickness of the interfacial layer and the shape of the density profile. Some of these discrepancies might be partly due to the experimental methods employed. The use of a shadowgraph might be misleading in determining $h$ because the method averages over the entire width of the experimental facility at constant depth, which might be different from the time-averaged interfacial thickness. Moreover, we note that a travelling conductivity probe may produce significant perturbations as it penetrates the density interface.

In experiments using different types of solutes, Wolanski \& Brush (1975) observed that $n$ is a function of $P_{r}$, where $P_{r}$ is the Prandtl number given by $P_{r}=\nu / \kappa$, and $\nu$ 
is the kinematic viscosity. This is equivalent to a functional dependence on the Péclet number, since $v$ was not varied significantly. The observations of Wolanski \& Brush (1975) cannot be explained by either Linden's $(1973,1975)$ or Long's (1978) theoretical models, since both are independent of viscosity and diffusion effects (at sufficiently high Reynolds numbers). McDougall (1979) suggested that this observation might be due to non-Newtonian effects exhibited by some of the fluids used by Wolanski \& Brush (1975).

\subsubsection{Lou-Péclet-number investigations}

In experiments involving heat as a stratifying agent, Crapper \& Linden (1974) found that molecular diffusion is important in the determination of the interfacial structure at $P_{e} \leqslant 200$. In this case they found that a diffusive core is formed at the centre of the interfacial layer, across which all transport occurs by molecular diffusion. Fernando (1986) pointed out that if all transport is due to molecular diffusion then $u_{\mathrm{e}} \sim \kappa / h$. By invoking the relationship $h / l_{0} \sim P_{e}^{-\frac{1}{2}}$, a result in agreement with the findings of Crapper \& Linden (1974), Fernando obtained $u_{\mathrm{e}} /\left(\sigma_{u}\right)_{0} \sim P_{e}^{-\frac{1}{2}}$. This is clearly different from the $R_{i}^{-1}$ behaviour observed by Turner (1968). Denton \& Wood (1981) suggested that at sufficiently large $R_{i}, u_{\mathrm{e}} /\left(\sigma_{u}\right)_{\mathrm{o}} \sim$ $P_{e}^{-1}$ and that the $R_{i}^{-1}$ behaviour observed by Turner occurs in a transition between the $R_{i}^{-\frac{3}{2}}$ and the $P_{e}^{-1}$ regimes when $u_{\mathrm{e}} /\left(\sigma_{u}\right)_{0}$ is plotted against $R_{i}$.

\subsubsection{High-Richardson-number range}

At very large $R_{i}$, Turner (1979) suggested that all mixing curves flatten out and become independent of $R_{i}$. Fortescue \& Pearson (1967) showed that at very large $R_{i}, u_{\mathrm{e}} /\left(\sigma_{u}\right)_{0} \sim P_{e}^{-\frac{1}{2}}$ in the context of gas absorption into a water surface. Turner (1979) suggested that their explanation of the $P_{e}^{-\frac{1}{2}}$ behaviour using a theory based on molecular diffusion into the large eddies of turbulence in the water, also seems appropriate for a very stable liquid-liquid interface. The $P_{e}^{-\frac{1}{2}}$ dependence is equivalent to stating that $u_{\mathrm{e}} \sim \kappa / h$, with $h$ given by $h \sim\left[\kappa \tau_{0}\right]^{\frac{1}{2}}$ and $\tau_{\mathrm{o}}$ being the integral timescale of the turbulence.

To obtain $u_{\mathrm{e}} /\left(\sigma_{u}\right)_{0} \sim P_{e}^{-1}$ at sufficiently high $R_{i}$ when the entrainment is governed by molecular diffusion, Denton \& Wood (1981) used $u_{\mathrm{e}} \sim \kappa / h$ together with $h \sim l_{\mathrm{o}}$ as observed by Crapper \& Linden (1974) at high $P_{e}$ with the stratification due to salt, Denton \& Wood also suggested that their model could be improved by allowing for a change in the interface thickness at low $P_{e}$ as observed by Crapper \& Linden. A consequence of such an improvement implies that the rate of entrainment changes from $P_{e}^{-\frac{1}{2}}$ to $P_{e}^{-1}$ as $P_{e}$ increases for sufficiently high $R_{i}$.

As evident from the above discussion there is a considerable amount of uncertainty in characterizing the nature and the dynamics of interfacial mixing processes. In particular, the mechanisms leading to the instability of the interface and subsequent mixing, as well as the turbulence-internal wave interactions, are not very well understood. In this paper, we report on the details of an investigation to study the nature of turbulent density interfaces by measuring the properties of the internal wavefield (amplitude, velocities and spectra) and characterizing the mechanisms that lead to wave breaking and mixing. Moreover, we measure the interfacial layer thickness, the vertical mass flux, and discuss the evolution of intermittent mixing events. Concentration measurements along a straight line were obtained by using laser-induced fluorescence (LIF) in conjunction with a linear photodiode array (with matching of refractive indices using salt and alcohol as solutes in the lower and upper 
layers respectively). The LIF technique is non-intrusive and is capable of providing both temporal and spatial resolution unattainable by conventional methods. Additionally, a laser-Doppler velocimeter (LDV) system was used to measure the vertical velocity component at a point where the concentration was also measured. We also discuss, based on the results of our experiments, a simple model for turbulent mixing at a shear-free density interface.

\section{Experimental method}

The experiments described in this work consist of linear concentration measurements through a density interface as well as velocity-concentration correlations. The bulk of the experimental procedures and equipment used in these experiments were discussed in Hannoun, Fernando \& List (1988). We will confine our discussion here to the linear photodiode camera system.

\subsection{Laser-induced fluorescence system}

The method of measuring concentrations by LIF is becoming increasingly popular. In the last few years Papanicolaou (1984), Koochesfahani (1984) and Papantoniou (1986) among others have successfully used this technique to obtain further insight into the nature of mixing in turbulent flows. The technique, as used in this study, is based on the ability of Rhodamine 6G dye to fluoresce at wavelengths near $570 \mathrm{~nm}$ when exposed to a laser light beam having a wavelength of $514 \mathrm{~nm}$. For a range of dye concentrations, the intensity of the emitted light is proportional to the concentration of the dye. This provides a non-intrusive method for measuring concentrations. The details of the method of concentration measurement by LIF will not be discussed here but a comprehensive discussion on this subject can be found in Liu et al. (1977).

In our set-up the $514 \mathrm{~nm}$ line of a $2 \mathrm{~W}$ argon-ion laser (Spectra Physics model 265) is directed vertically downward by the use of a combination of front-surfaced mirrors. The beam then passes through a small glass window placed at the free surface as shown in figure 1 of Hannoun et al. (1988). Before it enters the water the beam also passes through a $200 \mathrm{~cm}$ converging lens that is placed appropriately so that the beam is of nearly uniform thickness of about $0.5 \mathrm{~mm}$ in the test section. A section of the laser beam was imaged by an array of 1024 photodiodes (EG \& G Reticon model RL1024G). The photodiode array is encased in an LC300A camera from EG \& G Corporation. This camera is about the size of, and its operation is analogous to, an ordinary photographic camera, with the exception that the film is replaced by the photodiode array and its associated electronic circuitry. The array diodes are in a single row with a centre-to-centre spacing of $25 \mu \mathrm{m}$. The aperture width of each pixel is $26 \mu \mathrm{m}$. The field of view of the camera depends on the lens selected and its distance away from the laser beam. The object resolution is therefore determined by the field of view. In our experiments a $50 \mathrm{~mm} f 1.8$ Nikon lens was used and the LC300A camera was supported on a rigid surveying tripod. The imaged laser beam passes through the centre of the tank and the camera was at a distance of $6580 \mathrm{~cm}$ away from the beam thus giving a spatial resolution of about $0.3-0.4$ $\mathrm{mm}$. The array was scanned by a sample-and-hold circuit controlled by a clock, which switches from diode to diode at an adjustable frequency $f_{\mathrm{a}}$. After scanning the last diode, there is an adjustable blanking period before sampling the first diode of the next scan. $f_{\mathrm{a}}$ was supplied by a signal generator and was in the range of 
$85-110 \mathrm{kHz}$. The frequency at which data are available from each pixel is given by $f_{\mathrm{a}} /$ count, where 'count' equals the number of array diodes (1024) plus the blanking period elock cycles (fixed at 64 in this study). This gives a data output rate of about $80-100 \mathrm{~Hz}$ per pixel. However, owing to the large amount of data generated and the limited magnetic disc storage volume, only a fraction of the available data was digitized and stored on the disk. Typically, we digitized every fourth scan thus yielding a frequency of about $20-25 \mathrm{~Hz}$ per pixel. Alternatively, we could have obtained these frequencies by using a smaller $f_{\mathrm{a}}$ and digitizing every scan. However, large values of $f_{\mathrm{a}}$ help in preserving the sharpness of the flowfield image. A total of 5120 array scans were recorded in each experiment. Details of the electronic circuitry are included in the Reticon LC300A manual (1981).

The analog output of the camera has an amplitude of zero to $1.0 \mathrm{~V}$. Dark noise is typically less than $10 \mathrm{mV}$, giving a dynamic range of $100: 1$. The analog output is digitized using a custom built A/D converter as discussed in Hannoun et al. (1988) (details are in Papantoniou 1986). The data acquisition software was written by Dr D. Papantoniou and used assembly language subroutines that maximized the efficiency of the process. The program also sampled two AD11-K analog-to-digital converter channels that were connected to the analog outputs of two LDV signal processors that measured a horizontal and the vertical velocity components. The AD11-K channels were sampled synchronously at the end of each array scan. Thus, velocities were also sampled at a rate of $20-25 \mathrm{~Hz}$. Other details of the data acquisition process are in Papantoniou (1986).

Before experiments to measure concentration were carried out, a calibration was performed in order to account for the effects of lens aberration, variable beam width, and various imperfections such as distortions caused by the glass windows of the tank. Rhodamine 6G dye was mixed with dechlorinated water in the tank thus yielding a homogeneous fluid with a known dye concentration. The beam was then focused onto the camera and the average output from 512 scans was recorded by the computer to provide a camera calibration output as a function of the pixel number. Subsequent to the calibration, special care was taken to ensure that the camera was not touched until the experiment was over. The dye concentration during a calibration was determined so as to be within $10 \%$ of the dye concentration in an actual experiment. This was needed since the emitted fluorescent light seemed to attenuate as it propagated through the fluid containing Rhodamine $6 \mathrm{G}$ on its way towards the photodiode array. It is to be noted that this kind of attenuation is different from the attenuation of the original incident laser beam as it passes through the Rhodamine $6 \mathrm{G}$ solution. Within the range of dye concentration used in this experiment, the attenuation of the emitted fluorescent light was estimated to be less than $10 \%$ of the light received by the array. Another precaution that was taken to reduce the effect of fluorescent light attenuation was the positioning of the camera at a higher vertical position than the density interface. This was needed to ensure that all the emitted fluorescent light incident on the array passed through a homogeneous solution, i.e. the upper mixed layer, and was therefore attenuated equally. By positioning the camera above the interface and using similar dye concentration values in both the calibrations and the experiments, errors due to the attenuation of the emitted fluorescent light are limited to less than $1 \%$ of the values recorded by the photodiode array.

After the data were recorded on magnetic media, they were transferred to a PDP 11/24 and a VAX 750 computers for data reduction. The LIF data were corrected by taking into account the previously obtained calibration curves. The data were 


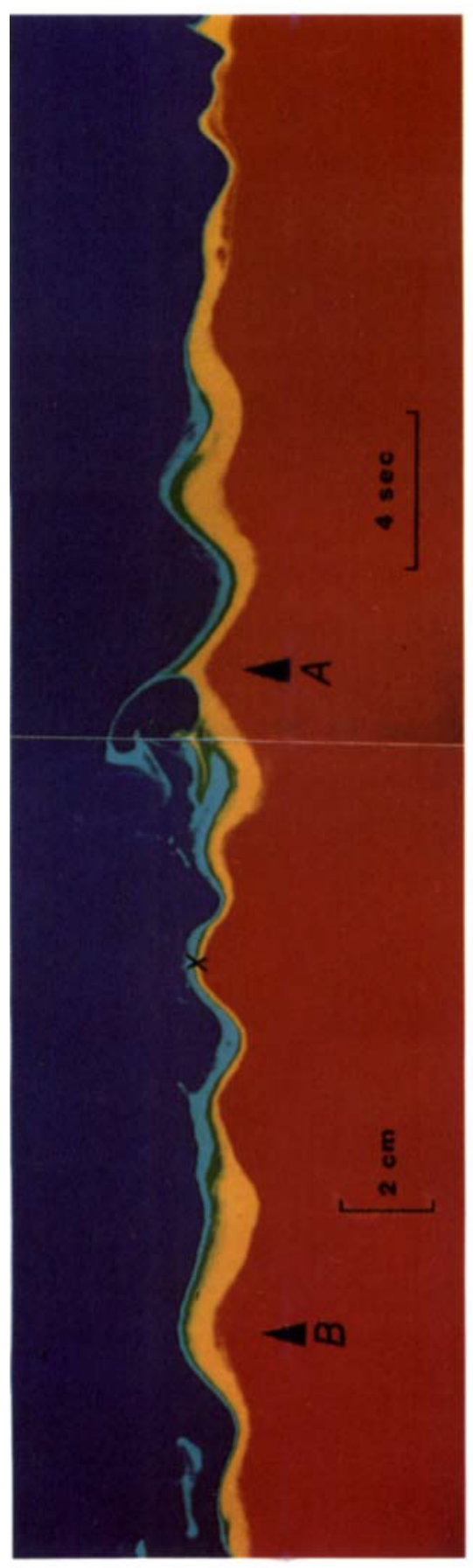

ฮ
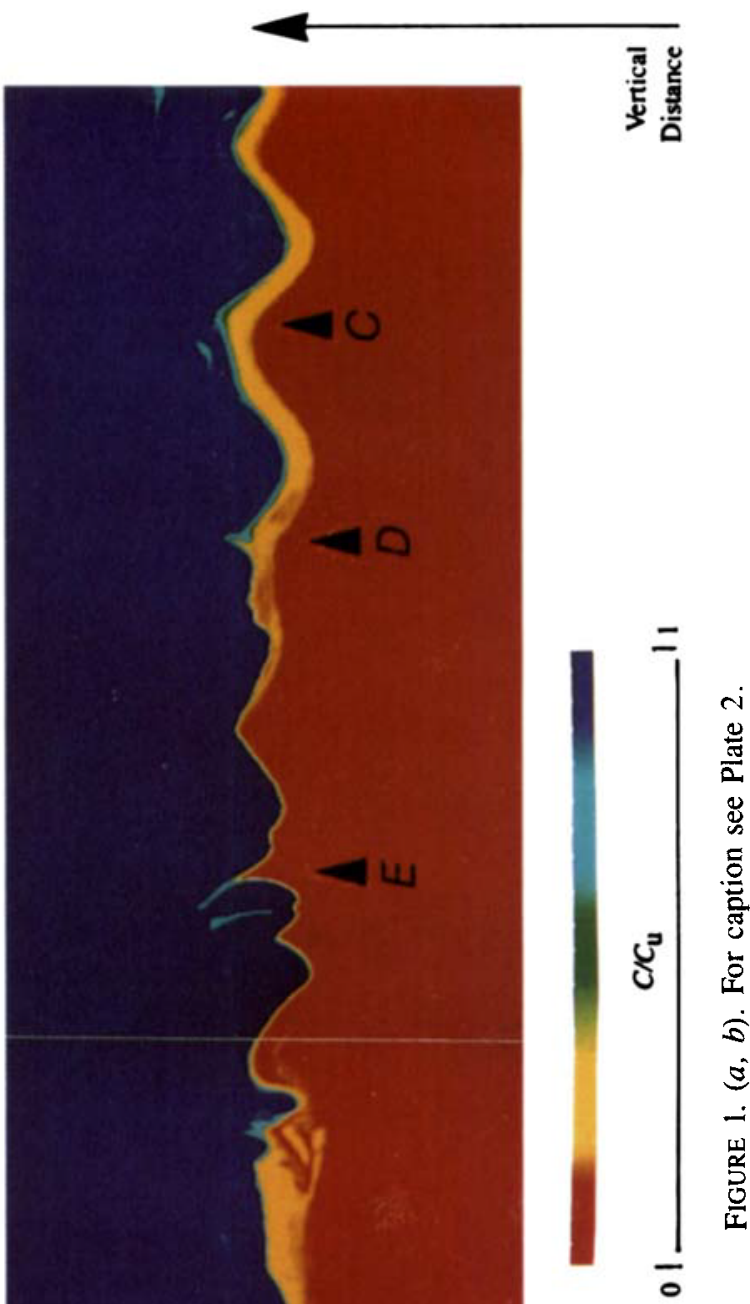


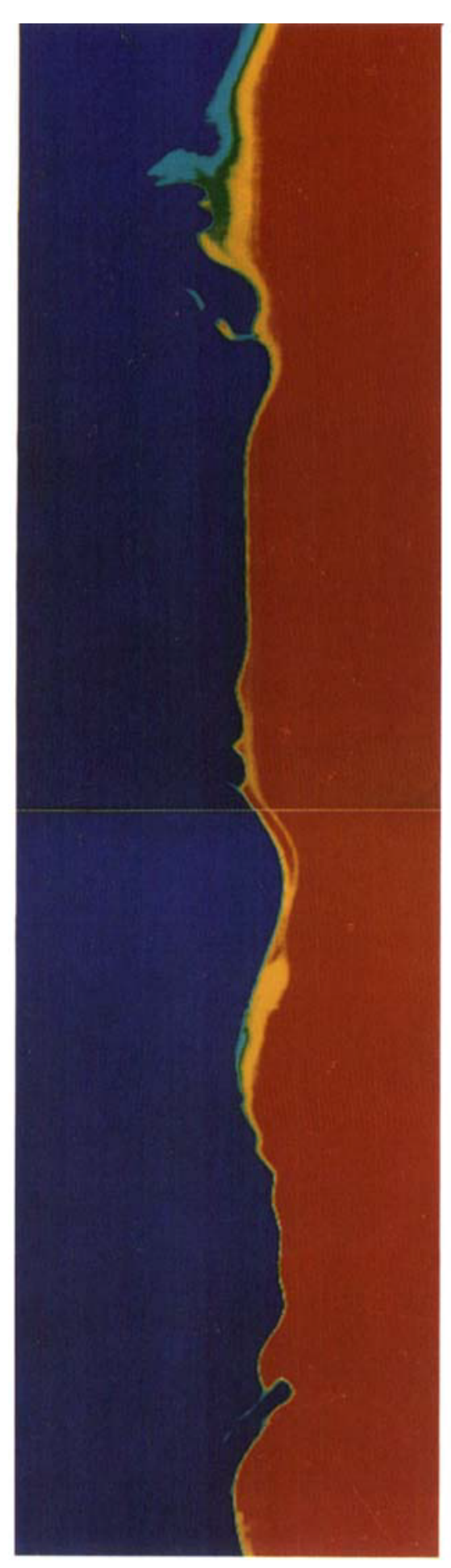

(ิ)

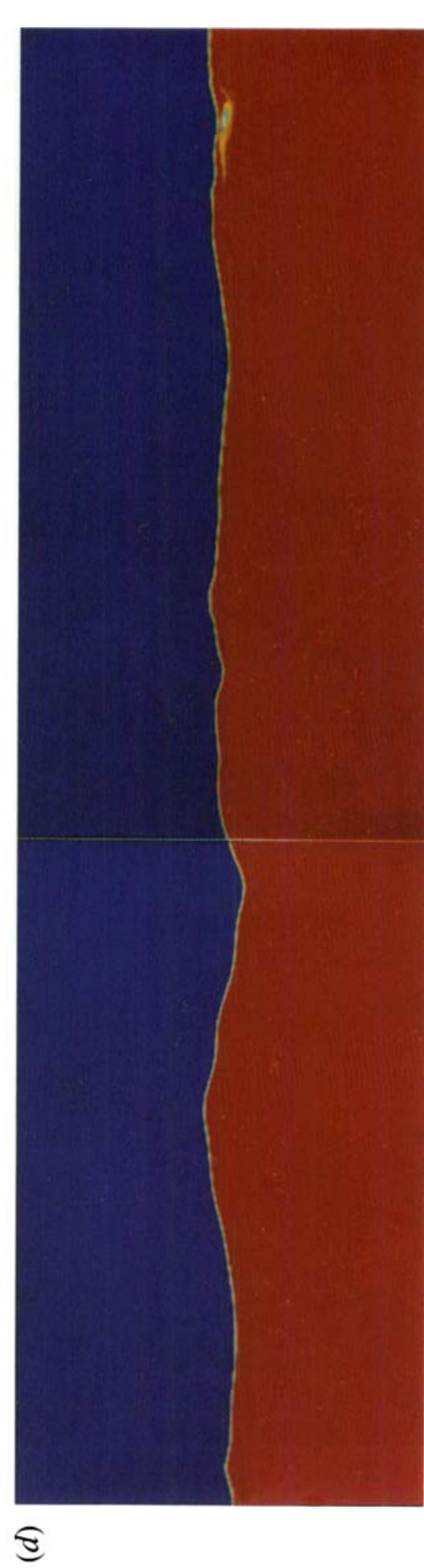

움

受

s.

苞

.매욤

政

言

응 궁

$\stackrel{8}{\square}$

$\infty$ 政

II $\frac{7}{3}$

os

छ.

iे

II $\bar{\pi}$

$\approx$

0

نे

$\| \dot{m}$

र्ट

電

S.

密

要药

U

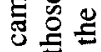

ธ응

号过

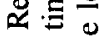

決

몰

은

包

马 용 로 它我 过 혼

政

萿

言㻤

压

-

एँ

喜 
also corrected for the attenuation of the laser beam as it passed through the Rhodamine $6 \mathrm{G}$ solution by using a relationship of the form:

$$
I=I_{0} \exp \left(-\int_{0}^{x} \epsilon C \mathrm{~d} x\right)
$$

where $I$ is the intensity of the laser beam after it traverses a distance $x$ in the fluid, $I_{0}$ is the initial intensity of the laser beam at $x=0, C$ is the dye concentration, and $\epsilon$ is the dye absorption coefficient (constant for the range of dye concentrations used in this study). The laser beam attenuation is actually determined by replacing the integral in the last equation with a summation and using the values of $C$ as obtained from the array output. The details of the method of correction for attenuation are discussed in Koochesfahani (1984). The maximum correction due to beam attenuation was kept to below $20 \%$ by using sufficiently low dye concentrations. Moreover, owing to the fact that the dye concentration away from the interface is almost constant, large errors resulting from miscalculating the attenuation can be detected. The constant $\epsilon$ was determined experimentally by recording the fluorescent light intensity after the beam traversed a measured distance in several solutions with different but known dye concentrations. The value of $\epsilon$ was found to be $300 / \mathrm{cm} \mathrm{g} / \mathrm{l}$ and details of its method of determination are found in Hannoun (1987). Other details of the data reduction process are in Papantoniou (1986).

\section{Preliminary observations}

The most convenient and illustrative method of presenting the linear concentration data is by displaying them in false colour images using a colour video screen and an image processor. Figure $1(a-d)$ (Plates 1 and 2) presents such images of the flow reconstructed from the Reticon camera data. In this figure, the vertical axis represents a vertical distance whereas the horizontal axis represents time. The concentration data, which have values from 0 to 255 (with 0 corresponding to fluid with no Rhodamine 6G dye), are colour coded according to the spectrum accompanying figure 1 . It is worth noting that $C$ is the tracer concentration and $C_{\mathrm{u}}$ is the average concentration in the upper mixed layer. The form of the Richardson number that will be used in interpreting the data is $R_{j}=\Delta b L_{0} /\left[\sigma_{u}\right]_{0}^{2}$ where [ ] denotes a spatial average in a horizontal plane and $L_{\mathrm{o}}$ is an integral lengthscale of the turbulence. $L_{\mathrm{o}}$ is determined as $L_{\mathrm{o}}=\left[\sigma_{u}\right]_{\mathrm{o}}[\tau]_{\mathrm{o}}$ where $[\tau]_{\mathrm{o}}$ is the integral timescale of the turbulence defined in this study as the area under the vertical velocity autocorrelation coefficient curve. Moreover, the subscript $o$, here and subsequently, refers to measurements in homogeneous fluid at the vertical location of the interface. Note that this definition of the Richardson number differs from $R_{i}$ used by other investigators only in the definition of the integral lengthscale $L_{0}$ and in the fact that the turbulent velocity variance used is a spatial average over a horizontal line rather than that measured at a single point. We experimentally determined that $L_{0}=0.1 D$ where $D$ was measured from a virtual origin coinciding with the lowest point during the grid displacement cycle. Owing to the transient nature of the experiments, $D$, and hence $R_{j}$, are slowly increasing functions of time. However, the maximum change in $D$ in any one experiment was limited to less than $13 \%$ of its original value (and much less than that at higher $R_{j}$ ). $R_{j}$ was based on average values during each experiment and was in the range $24-98$.

While concentration profiles along a vertical line do not provide clear information regarding the horizontal structure of the fluid motion, they reveal some striking 


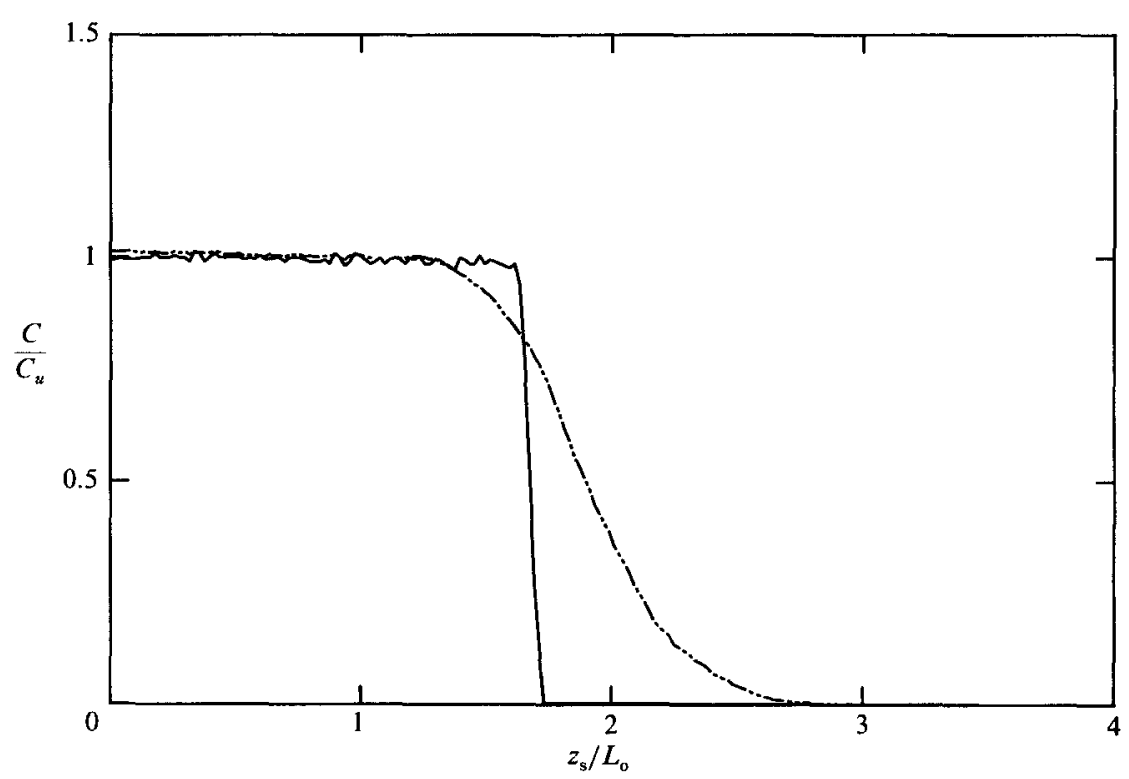

Figure 2.-.-, $30 \mathrm{~s}$ time-averaged concentration profile. - - ' instantaneous' concentration profile obtained in less than $10 \mathrm{~ms} . R_{j}=24$.

features of the interfacial structure. In figures $1(a)$ and $1(b)$, which present sequential flow images at $R_{j}=24$, it can be seen that the amplitude of the interfacial waves $\dagger$ is of the same order of magnitude as the thickness of the interfacial layer. We can also observe that the shape of the concentration profile varies with time and cannot be adequately described by any one specific profile. In particular, an instantaneous concentration profile does not bear much resemblance to the average concentration profile, a result which is in disagreement with Crapper \& Linden (1974). Figures $1(a)$ and $1(b)$ also indicate that the interfacial waves seem to break intermittently thus giving rise to localized patches of turbulent fluid. This breaking of the internal waves seems to occur predominantly at the crests of the waves (i.e. when the interface is near its highest point of oscillation). After the occurrence of these local instabilities, some mixed fluid is transported upwards and ultimately becomes fully incorporated into the upper mixed layer. As $R_{j}$ increases, as shown in figures $1(c)$ and $1(d)$, the amplitude of the interfacial waves as well as the thickness of the interfacial layer tend to decrease. Additionally, the interface seems to get 'calmer' and the frequency of violent overturns decreases with increasing $R_{j}$. At $R_{j}=98$ as shown in figure $1(d)$, the mixing events seem less frequent and not as violent as at lower $R_{j}$. However, this should be regarded with caution, since in this case the thickness of the interfacial layer is comparable with the spatial resolution of our equipment. As a result, some mixing events might go undetected.

In figure 2 , a concentration profile at $R_{j}=24$ and time-averaged over $30 \mathrm{~s}$ is shown, where $z_{\mathrm{s}}$ is a distance from an arbitrary origin. An 'instantaneous' concentration profile obtained in less than $10 \mathrm{~ms}$ is also presented. We can observe, in a more quantitative way than in figure 1, that the instantaneous concentration profile does not bear much resemblance to the average profile. This is thought to be due to both (i) the contribution of internal waves to the average thickness of the

+ It is not obvious that the interfacial displacements are in fact waves, but given that the interface only 'breaks' occasionally, we refer to the disturbances as waves. 


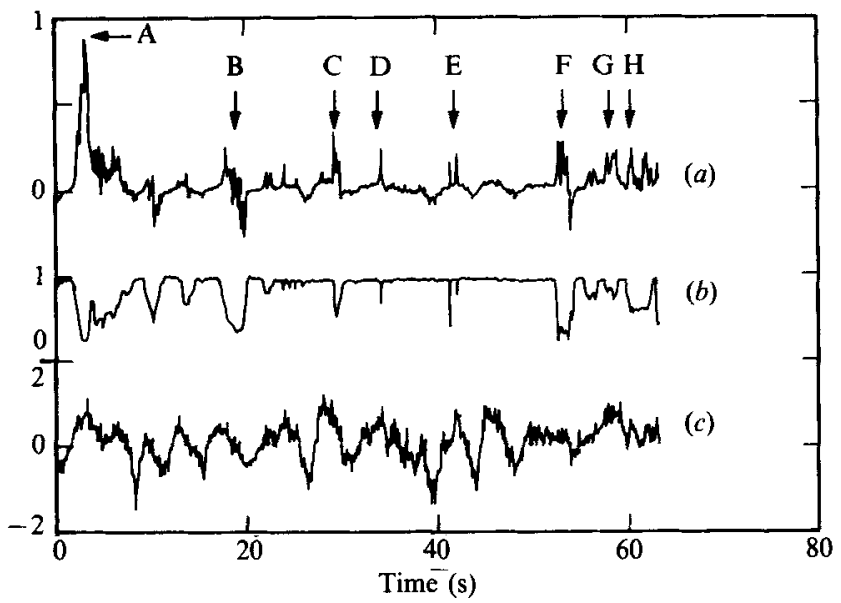

Figure 3. Time history of the vertical velocity, concentration, and the vertical flux of clear fluid as measured at the vertical location $\times$ in figure 1 . $(a)$ vertical velocity $w$ in $\mathrm{cm} / \mathrm{s} v s$. time. $(b) C /$ $C_{\mathrm{u}}$ vs. time. (c) Vertical flux of clear fluid $\psi(\mathrm{cm} / \mathrm{s})$ vs. time. The events marked A-H correspond to those marked in figure $1(a, b) . R_{j}=24$.

interfacial layer and to (ii) the temporal change in the shape of the instantaneous profile. The significance of the internal wave motions in determining the timeaveraged concentration profile depends on the ratio of the amplitude of the internal waves to the instantaneous thickness of the density interface. The larger this ratio, the more important the interfacial wave contribution will be. It will be seen from results in $\$ 4$ that this ratio increases with increasing $R_{j}$. The temporal change in the shape of the instantaneous profile is determined by the dynamies of the interface and the occurrence of instabilities and mixing events. It can be observed from figure 1 that the temporal variability in the shape of the instantaneous concentration profile decreases with increasing $R_{j}$. Quantitative results concerning the thickness of the interface and the properties of the internal waves will be presented in $\S 4$.

It is of interest to investigate further the intermittent mixing events within the density interface. Figure 3 presents a time history of the vertical velocity component $w$ at a point near the interface for the same experiment depicted in figures $1(a)$ and $1(b)$. The vertical position of the point at which the velocity was measured is marked on figure $1(a)$. Moreover, the normalized dye concentration $C / C_{\mathrm{u}}$ at that same point is also shown. Another quantity that is presented in figure 3 is $\left.\psi=\left[C-C_{\mathrm{u}}\right) / C_{\mathrm{u}}\right] w$ which represents the vertical flux of the clear (lower) fluid. In this particular case, $\psi$ is more useful as an indicator of interfacial activity than $\left(C / C_{\mathrm{u}}\right) w$, the vertical dye flux. This is a result of $\psi$ preferentially disclosing motion of lower fluid mixing into the upper layer fluid as shown in figure 3 . The temporal relationship between figure 3 and figure $1(a, b)$ is established by the use of alphabetically labelled arrows that correspond to the same instances in time.

Event A depicted in figure 1(a) indicates that some lower-layer fluid is being ejected upwards. An examination of figure 3 indicates that at $A$, the vertical velocity maintains significant upward (positive) values. Figure 3 also shows that $\psi$ remains positive for about $5 \mathrm{~s}$. These observations imply that event A represents a situation where there is a net vertical mass flux and can hence be labelled a mixing event. On the other hand, $\psi$ in event $B$ switches signs going from positive to negative. The net vertical mass flux associated with $B$ is small. This implies that the observed 
behaviour at $B$ is due to an interfacial wave since the net vertical mass flux associated with internal waves is zero. An examination of figure $1(a)$ also supports this observation.

Other occurrences of events leading to a net vertical mass transport can be determined from an examination of $\psi$ in figure 3 . There are several discrete positive peaks in $\psi$ without accompanying comparable negative values. The arrows labelled $\mathrm{C}, \mathrm{D}, \mathrm{E}, \mathrm{G}$ and $\mathrm{H}$ all indicate such events. It can also be observed from figure 3 that the vertical velocity maintains significant upward values for these events. The length of time during which $w$ is upward is usually longer than the time $\psi$ remains positive for the individual events. The flow images at $\mathrm{E}$ and $\mathrm{G}$ in figure $1(b)$ clearly reveal that some fluid is being ejected whereas the processes involved at $\mathrm{C}, \mathrm{D}$ and $\mathrm{H}$ are less obvious. However, we can see that at $\mathrm{C}, \mathrm{D}$ and $\mathrm{H}$, the interfacial structure is abrupt and pointed and does not exhibit the smoothness associated with relatively uninterrupted internal waves (such as B). It can also be observed that at C, D and $H$, the breakdown of the interfacial waves occurs at the crests. Furthermore, the abruptness of the concentration contours for these events is most obvious near the top of the interfacial layer. In particular, events $\mathrm{C}$ and $\mathrm{H}$ indicate that the lower part of the interfacial layer (displayed in yellow and red) remains virtually unaffected by the wave breakdown.

The events marked $\mathrm{A}$ and $\mathrm{E}$ have some similarities in that they both clearly reveal lower-layer fluid being scoured upward, and also exhibit relatively large slopes of the concentration contours. However, the thickness of the interfacial layer prior to these events is markedly different. The images at these locations suggest that an interfacial instability occurs at $A$ and $E$, with a lengthscale of the order of the interfacial thickness just prior to the occurrence of the events. The event marked $\mathbf{F}$ indicates that $\psi$ switches sign from positive to negative. The net flux of clear fluid associated with $\mathbf{F}$ is upward since the net area under the $\psi$ curve in the vicinity of $\mathbf{F}$ is positive. All these observations, when viewed in their entirety, indicate that the intermittent mixing events occur sporadically in time.

\section{Characteristics of the interface and the internal wave field}

In this section we will present some quantitative results concerning the dynamics of the density interface and the internal wave field. Figure 4 presents concentration contours of $C / C_{\mathrm{u}}$. Three contours are shown corresponding to $C / C_{\mathrm{u}}=\alpha$ with $\alpha$ taking the values $0.1,0.5$ and 0.9 . To obtain a certain $C / C_{\mathrm{u}}=\alpha$ contour the following procedure was followed: (i) for each array scan determine, starting from the bottom of the scan, the vertical position of the first point at which $C / C_{\mathrm{u}}$ exceeds $\alpha$ (ii) repeat (i) for every scan (iii) plot the vertical position versus time. One consequence of the use of this method for determining contours is its inability to determine multiple occurrences of $C / C_{\mathrm{u}}=\alpha$ in the event of concentration inversions. However, the fraction of time when there is an inversion in the concentration profile is small in all our experiments.

Contours similar to the ones presented in figure 4 are very useful in studying the dynamics of the density interface. The slope of a least-squares straight line fitted to a particular contour is a measure of the vertical velocity $u_{\mathrm{e}}$ of the interface. Such least-squares lines were obtained and a representative one is shown in figure 5 . The results indicate that $u_{\mathrm{e}} /\left[\sigma_{u}\right]_{0} \sim R_{j}^{-n}$ with $n$ being close to $\frac{3}{2}$, as shown in figure 6 . This result is in agreement with Hopfinger \& Toly (1976) and Turner (1968). The magnitude of the deviations of a certain contour from the least-square straight line, 


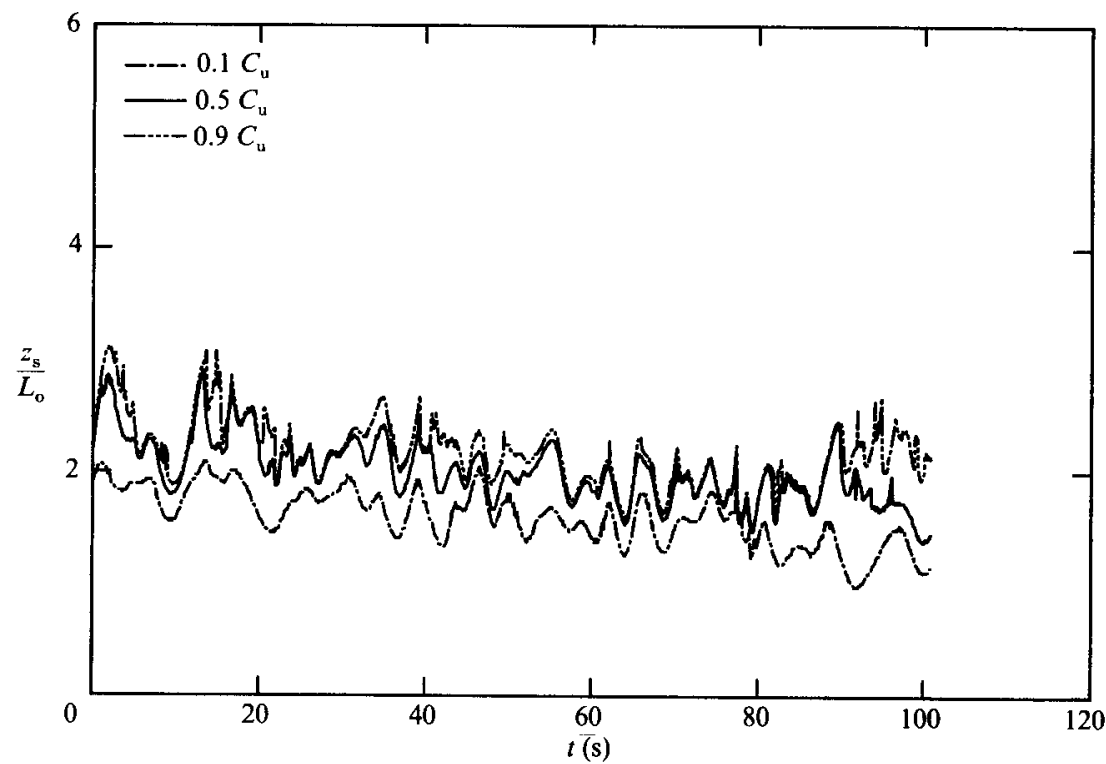

Figure 4. Concentration-time contours corresponding to $C / C_{\mathrm{u}}=0.1,0.5$ and $0.9 . R_{j}=24$.

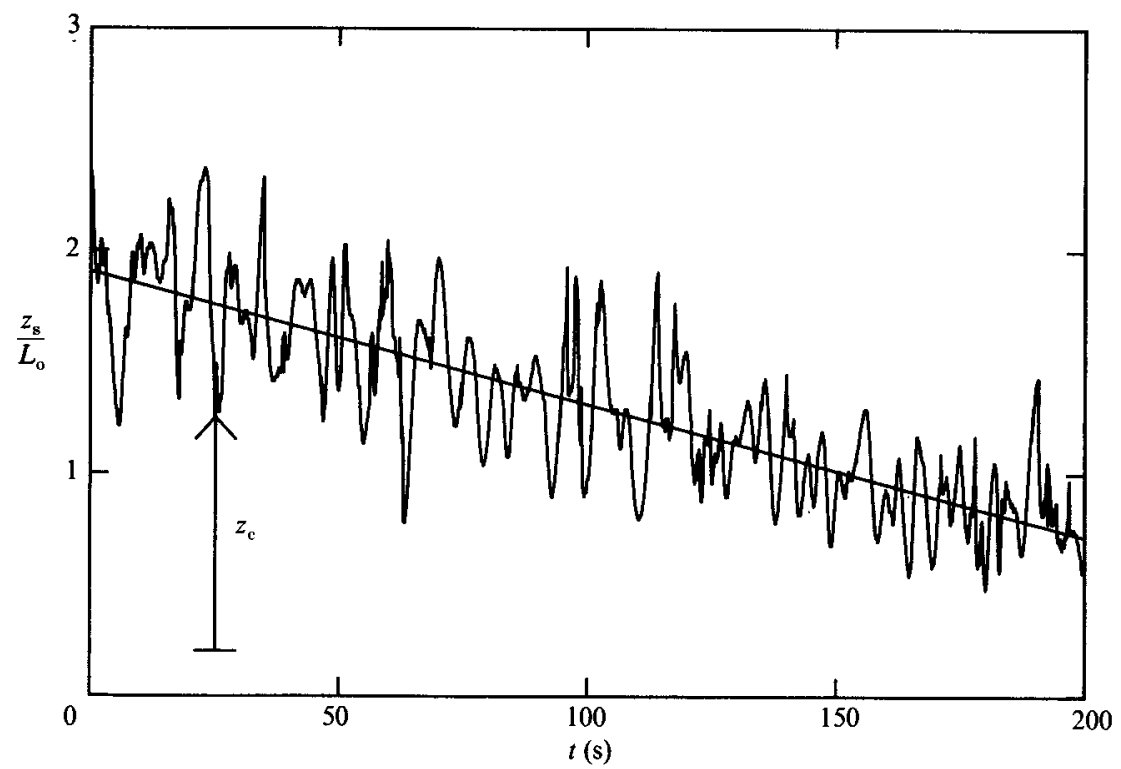

Figure 5. Concentration-time contour for $C / C_{\mathrm{u}}=0.5$ at $R_{j}=24$. The straight line is a leastsquares best fit for the contour.

lenoted by $\chi$, yields a measure of the amplitude of the interfacial waves. To letermine a representative quantity that characterizes the amplitude of the internal waves we located the $C / C_{\mathrm{u}}=0.1,0.5$ and 0.9 concentration contours for each sxperiment and then determined the least-squares best fit line for each of these sontours. The r.m.s. value of $\chi$ was then computed and its average for the three lifferent contours in each experiment, denoted by $\zeta$, was determined. $\zeta$ is an overall neasure of the amplitude of the internal waves. The averaging procedure was used jo compensate for the limited length of record available in each experiment. 


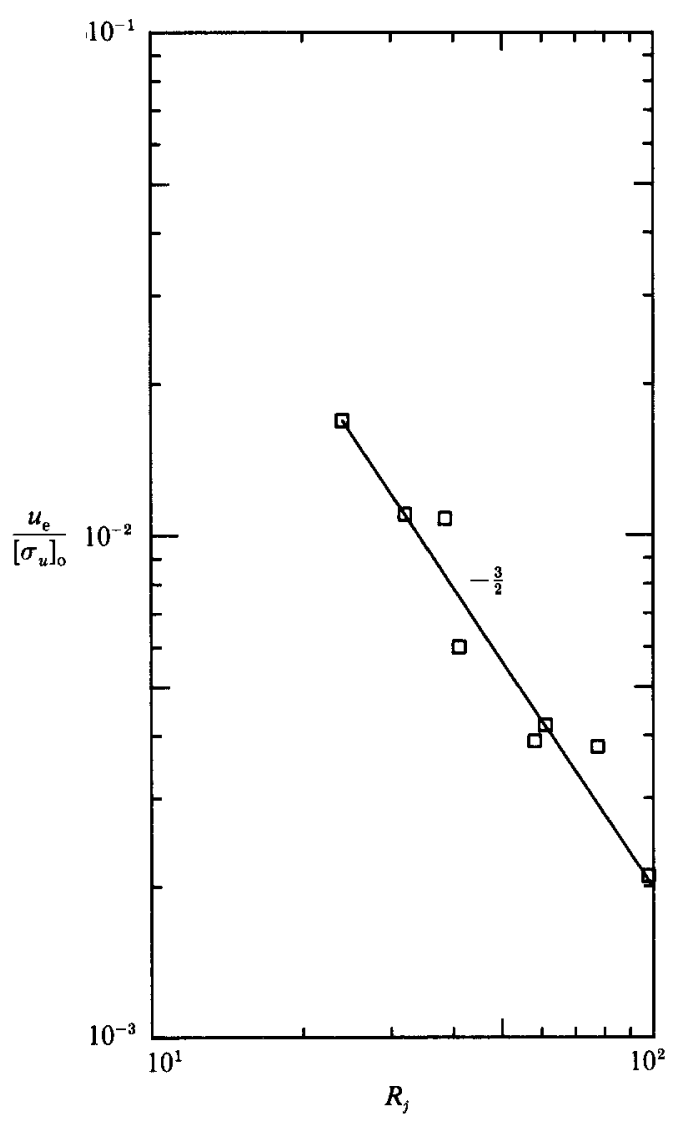

Figure 6. Variation of the normalized entrainment rate with the overall Richardson number $R_{j}$.

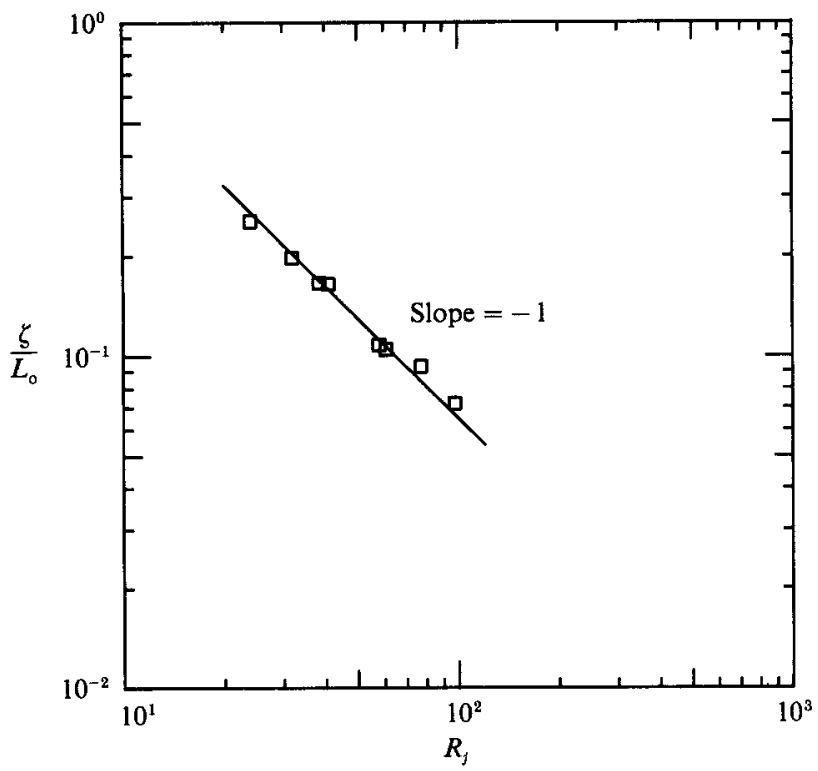

Figure 7. Variation of the normalized internal wave amplitude $\zeta / L_{0}$ with the overall Richardson number $R_{i}$. 


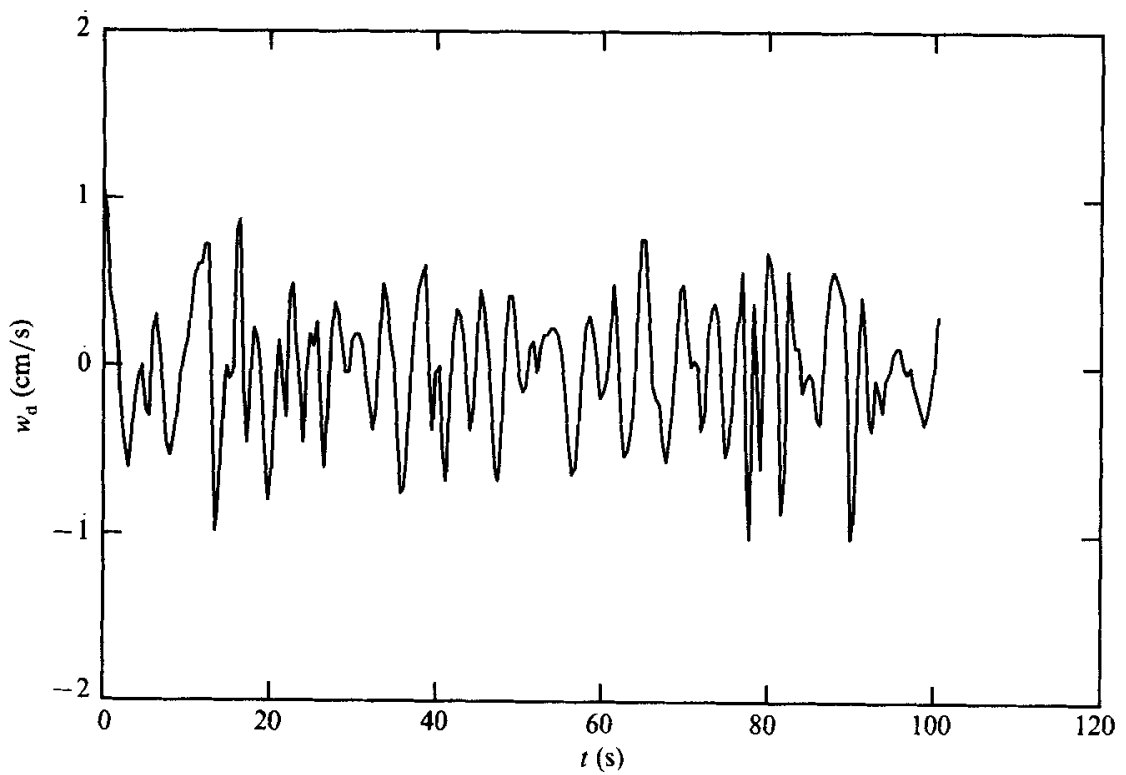

Figure 8. Variation of the instantaneous vertical wave velocity $w_{d}$ with time. The record is obtained from the $C / C_{\mathrm{u}}=0.5$ contour at $R_{j}=24$.

Figure 7 presents a plot of $\zeta / L_{\mathrm{o}}$ versus $R_{j}$. It can be seen that a relationship of the form $\zeta / L_{0} \sim R_{j}^{-1}$ provides a good fit for the experimental data. Actually, a leastsquares line fit to the data yields $\zeta / L_{\mathrm{o}} \sim R_{j}^{-0.91}$. An $\zeta / L_{0} \sim R_{j}^{-1}$ relationship implies that $\zeta \sim\left[\sigma_{u}\right]_{o}^{2} / \Delta b$, i.e. the amplitude of the interfacial displacements (waves) is determined by a balance between the kinetic energy of the eddies and the buoyancy jump across the density interface.

If $z_{\mathrm{c}}$ is a vertical distance to a certain contour line measured from an arbitrary origin as shown in figure 5 , then $w_{\mathrm{d}}=\mathrm{d}\left(z_{\mathrm{c}}\right) / \mathrm{d} t$ represents an instantaneous vertical velocity of the internal waves. Figure 8 presents $w_{\mathrm{d}}$ as a function of time obtained from the $C / C_{\mathrm{u}}=0.5$ concentration contour at $R_{j}=24$. Every tenth point in the original concentration contour was used and the derivative was calculated by taking the difference between two adjacent points. No smoothing or filtering was applied. The r.m.s. value of $w_{\mathrm{d}}$ was determined and the average of the r.m.s. values from the $C / C_{\mathrm{u}}=0.1,0.5$ and 0.9 contours, referred to as $w_{\mathrm{i}}$, was then computed. Here again, the averaging procedure was used to compensate for the limited time record in the experiments. $w_{i}$ is a measure of the vertical velocity of the internal waves. Figure 9 presents $w_{i} /\left[\sigma_{u}\right]_{0}$ as a function of $R_{j}$. It can be observed that $w_{\mathrm{i}}$ is well represented by a relationship of the form $w_{\mathrm{i}} /\left[\sigma_{u}\right]_{0} \sim R_{j}^{-\frac{1}{2}}$. After the determination of interfacial wave amplitudes and velocities, we obtained a measure of the internal wave frequency $n_{\mathrm{a}}$ defined as $n_{\mathrm{a}}=c_{1}\left(w_{\mathrm{i}} / \zeta\right)$ where $c_{1}$ is a constant. By invoking the $w_{\mathrm{i}} /\left[\sigma_{u}\right]_{\mathrm{o}} \sim R_{j}^{-\frac{1}{2}}$ and $\zeta / L_{\mathrm{o}} \sim R_{j}^{-1}$ relationships we obtain $n_{\mathrm{a}} \sim\left(\Delta b / L_{\mathrm{o}}\right)^{\frac{1}{2}}$. It is to be noted here that if most of the energy resides in the lowest internal mode, as is usually the case when the thermocline is relatively sharp (Phillips 1977), then $n_{\mathrm{a}}$ is approximately equal to the frequency of the lowest internal wave mode.

The vertical concentration gradient in each scan was determined by evaluating the spatial derivative of the concentration profile. The derivative was computed by taking the difference in concentration as defined by adjacent pixels. The maximum value of the vertical concentration gradient was obtained and its average over 


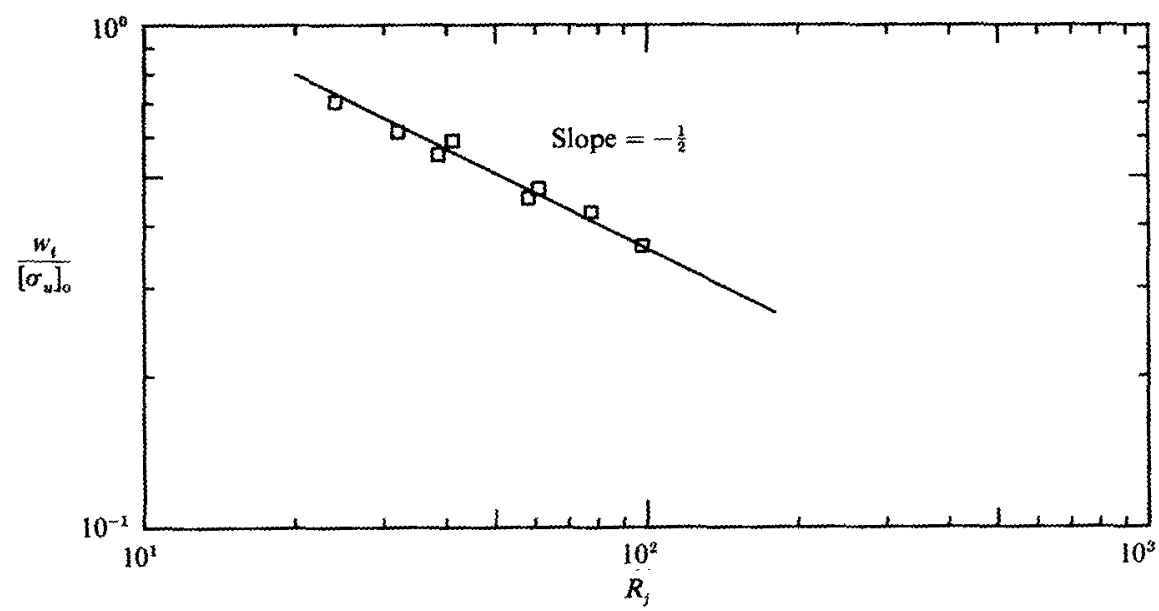

Fuvre 9. Variation of $w_{i} /\left[\sigma_{u}\right]_{0}$ with the overall Richardson number $R_{j}$.

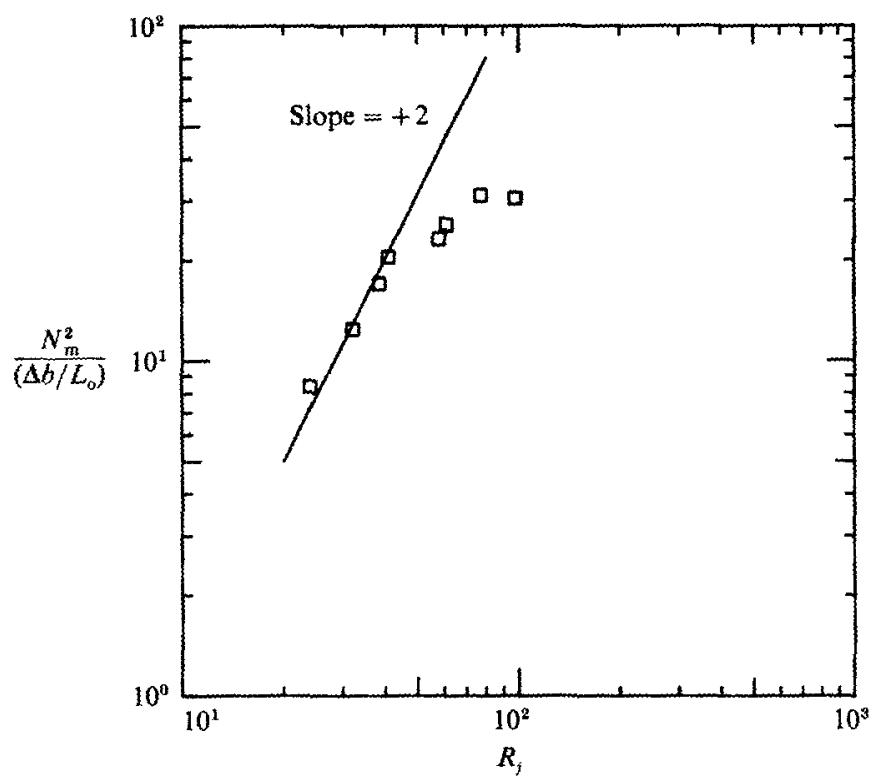

FIGURe 10. Variation of the normalized maximum Brunt $-V$ äisälä frequency $N_{\mathrm{m}}^{2} /\left(\Delta b / L_{0}\right)$ with the overall Richardson number $R_{j}$.

time, denoted by $\overline{C^{\prime}}$, was computed. The maximum average Brunt-Väisälä frequency, $N_{\mathrm{m}}$, in each experiment was computed using $N_{\mathrm{m}}^{2}=\left(\overline{C^{\prime}} / C_{\mathrm{u}}\right) \Delta b+\dagger$ A plot of $N_{\mathrm{rn}}^{2} /\left(\Delta b / L_{\mathrm{o}}\right)$ versus $R_{j}$ is shown in figure 10 . It can be seen that the data for $R_{j}<50$ can be well represented by a relationship of the form $N_{m}^{2} /\left(\Delta b / L_{0}\right) \sim R_{j}^{2}$. At higher $R_{j}$ the interface becomes sharp enough so that the calculation of $\bar{C}^{\prime}$ becomes significantly affected by the limited spatial resolution. For example at $R_{j}=61$, the

I It is worth noting that the concentration and density gradients are essentially equivalent if molecular diffusion is not important. This is the case because the fluid density is an almost linear function of the solute concentration considering the very low solute concentrations used in this study (Weast 1976). As a result, the value of $N_{\mathrm{m}}$ based on the concentration gradient will be approximately equal to that obtained from using the density gradient when the above conditions are satisfied. 
calculated $\overline{C^{\prime}}$ equals $0.49 C_{\mathrm{u}} / \Delta z$ where $\Delta z$ is the spatial resolution of the camera, which was $0.038 \mathrm{~cm}$ in that experiment. We anticipate that the effects of limited spatial resolution become important when $\overline{C^{\prime}}$ is about $0.5 C_{\mathrm{v}} / \Delta z$. As a result, we can conclude that for $R_{j}>50$, we are underestimating $N_{\mathrm{m}}$ because of limited spatial resolution. Hence, the results for $R_{j}>\mathbf{5 0}$ should not be viewed with much confidence. They provide only a lower limit on $N_{\mathrm{m}}^{2} /\left(\Delta b / L_{\mathrm{o}}\right)$. If an interfacial layer thickness $h_{\mathrm{c}}$ is defined as $h_{\mathrm{c}}=C_{\mathrm{u}} / \overline{C^{\prime}}$, then the $N_{\mathrm{m}}^{2} /\left(\Delta b / L_{\mathrm{o}}\right) \sim R_{j}^{2}$ relationship would lead to $h_{\mathrm{c}} / L_{\mathrm{o}} \sim R_{j}^{-2}$.

The results of saline density profile measurements at high $P_{e}$ obtained by Crapper \& Linden (1974) indicates that $h / l_{\mathrm{o}}$ is constant with $R_{j}$. In Crapper \& Linden's experiments $h$ was determined by least-squares fitting a straight line to the central $50 \%$ of the data points (at least 20 points) in the interface and extending this line until it intersected the mean density in the upper and lower layers. In the instances when the interface is sufficiently sharp in our experiments, the determination of the interfacial layer thickness by a method similar to that of Crapper \& Linden is not possible. This is due to the fact that the number of points with concentrations between 0.25 and $0.75 C_{\mathrm{u}}$ is usually much less than 20 and can be as low as zero. This makes a direct comparison with the results of Crapper \& Linden impossible. Although there is some difference in the method used to calculate $h$ and $h_{\mathrm{c}}$, it is thought that these differences alone cannot account for the discrepancies in the observed dependance of $h / l_{\mathrm{o}}$ and $h_{\mathrm{c}} / L_{\mathrm{o}}$ on $R_{j}$. It is also worth noting that values of $h_{\mathrm{c}} / L_{\mathrm{o}}$ as small as 0.03 were observed in our experiments. This is much smaller than the $h / l_{0}=1-1.5$ values in Crapper \& Linden. Some of the discrepancies in the interfacial thickness measurements may be due to the difference in spatial resolution between the two experiments. Crapper \& Linden used a conductivity probe travelling at $5 \mathrm{~cm} / \mathrm{s}$ with a vertical resolution of $0.1 \mathrm{~cm}$. Since the probe was travelling the actual resolution exceeds $0.1 \mathrm{~cm}$ and depends on the frequency response of the instrument and the 'washing of the probe'. The vertical resolution in our experiments was in the range of $0.03-0.04 \mathrm{~cm}$.

It is to be noted that the $R_{j}$ range is limited in our experiments due to some practical considerations. At very small $R_{3}$ the interface migrates very quickly, and as a result, we are not able to obtain records that are long enough for statistical purposes at fairly constant $R_{j}$. High values of $R_{j}$ may be obtained by either increasing the density difference, increasing the depth of the upper layer, or decreasing the grid-oscillation frequency. The latter option was not used because the Reynolds number would have been substantially lowered. $\dagger$ Moreover, the density differences that were used were kept low so as to reduce the effects of refractive index fluctuations. The depth of the upper layer $D$ was kept to below $25 \mathrm{~cm}$ to eliminate the undesirable wall effects observed by Fernando \& Long (1983) at large $D$. Additionally, higher $R_{j}$ values were not used because the resolution of the equipment becomes a limiting factor for $R_{j}>50$.

\section{Entrainment model}

Phillips (1977) has investigated theoretically the evolution of infinitesimal disturbances at a sharp density interface in the ocean in the absence of mean shear. He studied a case when the Brunt-Väisälä frequency is appreciable only within a small depth surrounding the thermocline. The analysis was focused on the lowest 120.

$\dagger$ Reynolds numbers based on the integral lengthscale and turbulence intensity were about 
mode of the internal wave at the interface. For the case when the layer above the thermocline is only a small fraction of the local depth, Phillips showed that the frequency of the first mode internal wave $\omega$ is given by

$$
\omega^{2}=(\Delta b)[k /(1+\operatorname{coth}(k D))],
$$

where $k$ is a horizontal wavenumber and $D$ is the depth of the layer above the thermocline. In the special case that $k \sim 1 / D$ the above relationship reduces to $\omega^{2}=\Delta b / D$. This last relationship is equivalent to a result obtained in $\S 4$, namely $n_{\mathrm{a}}^{2} \sim \Delta b / L_{\mathrm{o}}$, if we take into account that $L_{\mathrm{o}}=0.1 \mathrm{D}$.

Phillips then investigated the degradation of the first internal wave mode and considered the possibility of occurrence of a dynamic instability in the thermocline where the maximum rate of shear $U_{\mathrm{m}}^{\prime}$ induced by the internal waves is maximum. He postulated that if the rate of shear induced by the lowest mode is large compared with the rate at which the basic flow changes, i.e. if $U_{\mathrm{m}}^{\prime} \gg \omega$, then it might be anticipated that the stability criteria developed for stratified shear flows (Miles 1961) would be relevant for the case of the lowest internal wave mode at a relatively sharp thermocline in the absence of mean shear. Motivated by the results of instability theory in stratified shear flows, Phillips investigated the possibility of occurrence of similar interfacial instabilities when the local gradient Richardson number $J<\frac{1}{4}$ for the case of the lowest internal wave mode at a sharp density interface. The local gradient Richardson number $J$, defined as $J=\left(N_{m} / U_{\mathrm{m}}^{\prime}\right)^{2}$, was calculated by Phillips for this case to be

$$
J=\left\{N_{\mathrm{m}} / \omega-\omega / N_{\mathrm{m}}\right\}^{-2} k^{-2} s^{-2},
$$

where $s$ is the amplitude of the thermocline displacement. When the wavelength of the internal wave is large compared with the thermocline thickness, i.e. $\omega \ll N_{\mathrm{m}}$, we obtain $J=\left(\omega / N_{\mathrm{m}}\right)^{2}(k s)^{-2}$. The condition $J=\frac{1}{4}$ yields a limiting slope

$$
(k s)_{\mathrm{m}}=2 n_{\mathrm{a}} / N_{\mathrm{m}} \quad\left(n_{\mathrm{a}} / N_{\mathrm{m}} \ll 1\right) .
$$

Phillips suggested that if the energy supply to the internal wave mode continues after the above limiting condition is attained, a local instability may develop, with a lengthscale of the order of the interfacial layer thickness, giving rise to a patch of intense, small-scale turbulence. Phillips added that the energy acquired (and then ultimately dissipated) by the turbulence is at the expense of the internal wave, so that an occasional local instability of this kind can restrict the magnitude of the internal waves in a similar fashion to the way breaking limits the growth of surface waves.

The possibility of the occurrence of such an instability in our experiments will be investigated next. To compare our experimental results with the theory we will take $s \sim \zeta$ since $\zeta$ is a measure of the amplitude of the interfacial waves. If we take $k \sim 1 / L_{0}, \dagger$ then it follows that $\omega \sim n_{\mathrm{a}}$ and $J \sim\left(L_{\mathrm{o}} / \zeta\right)^{2}\left(n_{\mathrm{a}} / N_{\mathrm{m}}\right)^{2}$. The assumption that $n_{\mathrm{a}} \ll N_{\mathrm{m}}$ was used and is valid because in our experiments $N_{\mathrm{m}} \sim 5 \mathrm{~Hz}$ whereas $n_{\mathrm{a}}$ as determined from visual observations of concentration contours is about $0.1 \mathrm{~Hz}$. A plot of $J_{i}$, defined by $J_{i}=\left(L_{\mathrm{o}} / \zeta\right)^{2}\left(n_{\mathrm{a}} / N_{\mathrm{m}}\right)^{2}$, versus $R_{j}$ is shown in figure 11 and indicates that $J_{i}$ is constant with $R_{j}\left(R_{j}<50\right)$. For $R_{j}>50$, the $N_{\mathrm{m}}$ values are significantly affected by the limited experimental spatial resolution as previously discussed, and as a result, we anticipate that the $J_{i}$ values are overestimated.

$\dagger$ The assumption that $k \sim 1 / L_{0}$ implies that the wavelength of the lowest internal mode is set by the integral lengthscale of the turbulence near the interface (or equivalently the depth of the upper layer). The consequences of this assumption will be discussed later. 


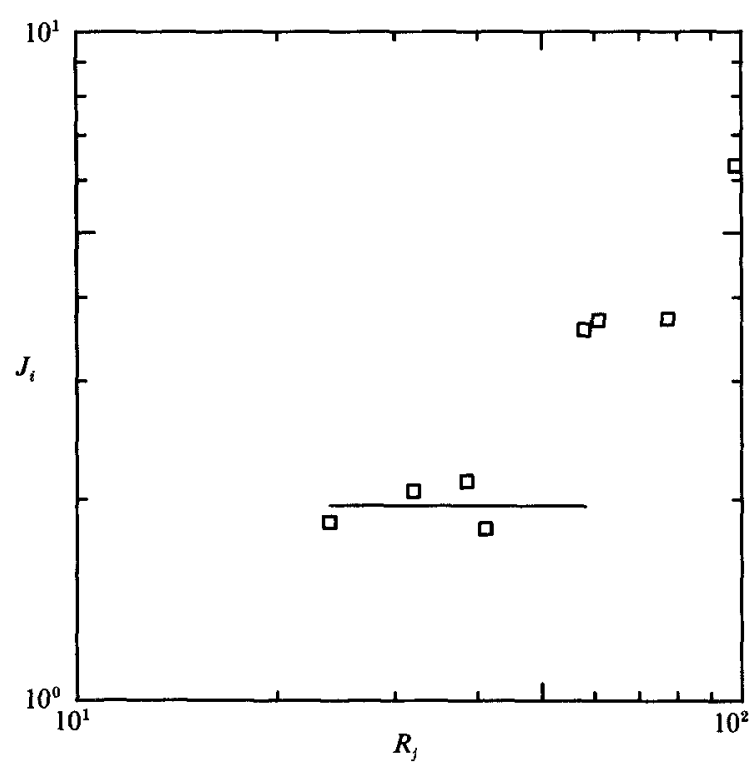

FigURE 11. Variation of the local gradient Richardson number $J_{i}$ with the overall Richardson number $R_{j}$. $\square$, experimental points :,$- J_{i}=1.96$.

Actually, if we use the results from $\S 4$, i.e. $\zeta \sim L_{0} R_{j}^{-1}$ and $N_{\mathrm{m}}^{2} /\left(\Delta b / L_{\mathrm{o}}\right) \sim R_{j}^{2}\left(R_{j}<50\right)$ we obtain $J_{i}=$ constant.

The facts that $n_{\mathrm{a}}^{2} \sim\left(\Delta b / L_{\mathrm{o}}\right)$ and $J_{i}=$ constant for a range of $R_{j}$, when viewed by themselves, may not provide sufficient evidence that an instability similar to the one discussed by Phillips (1977) is limiting the amplitude of the lowest-mode waves. To further test the applicability of Phillips' theory to our experiments, we will compare the theoretical predictions of the amplitude of the interfacial displacement and the internal wave spectra with our experimental results.

Phillips (1977) had calculated the mean square displacement of the thermocline $\overline{\alpha_{k}^{2}}$ when the process of local breakdown described above limits the amplitude of a single wavetrain with wavenumber $k$. He found

$$
\overline{\alpha_{k}^{2}}=2 n_{\mathrm{a}}^{2} /\left(N_{\mathrm{m}} k\right)^{2} .
$$

If most of the total energy resides in the lowest mode, as is usually the case when the thermocline is relatively sharp (Phillips 1977), then the internal wave amplitude is approximately determined by the mean square displacement of the thermocline due to the first mode. This implies that $s^{2} \sim \alpha_{k}^{\overline{2}}=2\left(\omega L_{\mathrm{o}} / N_{\mathrm{m}}\right)^{2} \sim L_{0}^{2} R_{j}^{-2}$ if we take $k \sim 1 / L_{\mathrm{o}}$ and $\omega \sim n_{\mathrm{a}}$ for the lowest internal mode. This result is equivalent to our experimental observation in $\S 4$, namely $\zeta / L_{0} \sim R_{j}^{-1}$, if we take $s \sim \zeta$.

If the lowest-mode waves are visualized as a random succession of wave groups at different wavenumbers, and if the growth of all wavenumbers is limited by an instability similar to the one discussed above, then the spectrum of the internal waves $\Phi(D, f)$ where $f$ is the frequency is predicted (Phillips 1977) to be

$$
\begin{aligned}
\Phi(D, f) \sim\left(D \Delta b N_{\mathrm{m}}^{-2}\right) f^{-1} & (f \ll \omega), \\
& \sim(\Delta b)^{2} N_{\mathrm{m}}^{-2} f^{-3} \quad\left(\omega \ll f \ll N_{\mathrm{m}}\right),
\end{aligned}
$$

where $\omega$ is the lowest-mode frequency. 


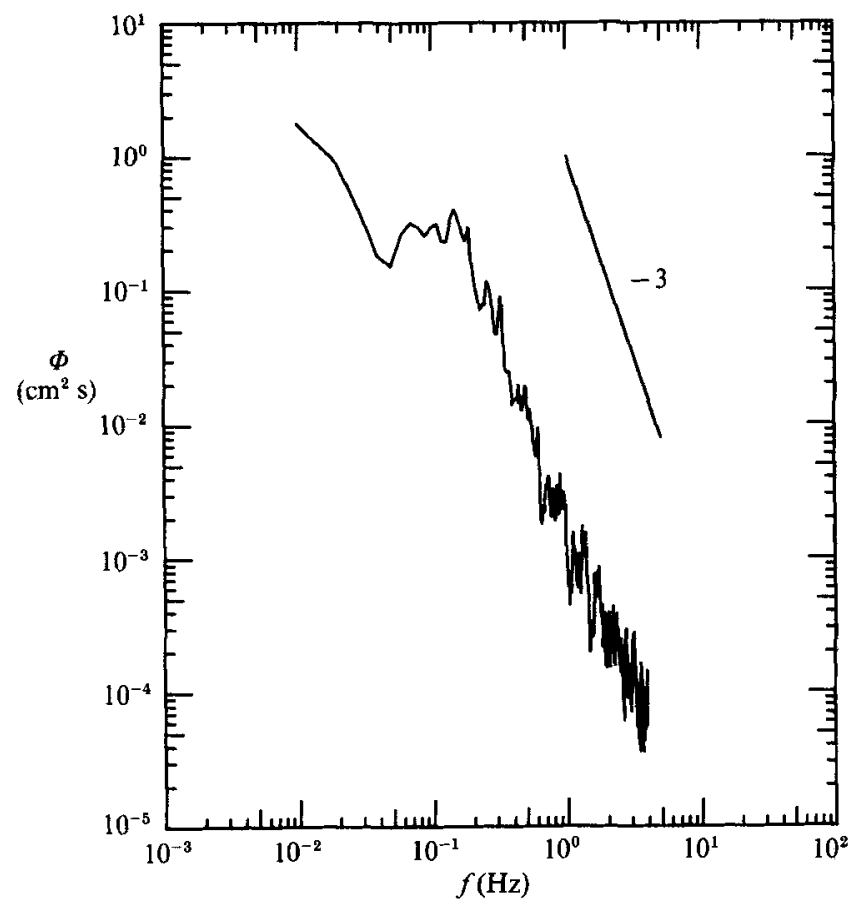

FigURE 12. Internal wave spectrum $\Phi$ at $R_{j}=24$.

An internal wave spectrum, obtained by a fast Fourier transform applied to the $C / C_{\mathrm{u}}=0.5$ contour at $R_{j}=24$ is presented in figure 12 . An $f^{-3}$ range is observed for a range where $n_{\mathrm{a}}<f<N_{\mathrm{m}}$. Moreover, spectra from different experiments, normalized by $(\Delta b)^{2} N_{\mathrm{m}}^{-2}$ with $N_{\mathrm{m}}^{2} \sim\left(\Delta b / L_{\mathrm{o}}\right) R_{j}^{2}$, are shown in figure 13 . All the spectra collapse thus giving support for Phillips' theory. If we use the actual measured $N_{\mathrm{m}}$ to normalize the spectra for $R_{j}>50$, the collapse is not as good. This might further indicate that $N_{\mathrm{m}}$ values in that range are significantly attenuated by the limited experimental spatial resolution.

The close agreement between our experimental results and the theory suggests the existence of a saturated wave field at the density interface and reveals that the process of local breakdown is limiting the growth of various wavenumber disturbances. The form of the spectra, the behaviour of the wave amplitude and frequency, as well as the constancy of $J_{i}$ with $R_{j}$, are all in agreement with the theoretical model. This agreement between the theory and the experiments, after invoking $k \sim 1 / L_{0}$, suggests that this relationship might be valid, thus implying that the wavelength of the lowest-mode internal waves is determined by the large turbulence scales. This is not surprising in view of the flattening of the large eddies near a density interface as observed in oscillating-grid experiments by Dickey et al. (1984) and Hannoun et al. (1988). As a result of this flattening, we might anticipate that the largest eddies in the mixed layer impose the largest horizontal lengthscale of the internal waves. The flow images shown in figure 1 also provide qualitative support for the above theory in that they show that the breakdown of the internal waves is a sporadic intermittent process. The images also seem to indicate that the lengthscale of the interfacial instability is comparable with the thickness of the interface. The events marked $\mathrm{A}$ and $\mathrm{E}$ in figures $1(a)$ and $1(b)$ clearly show that. 


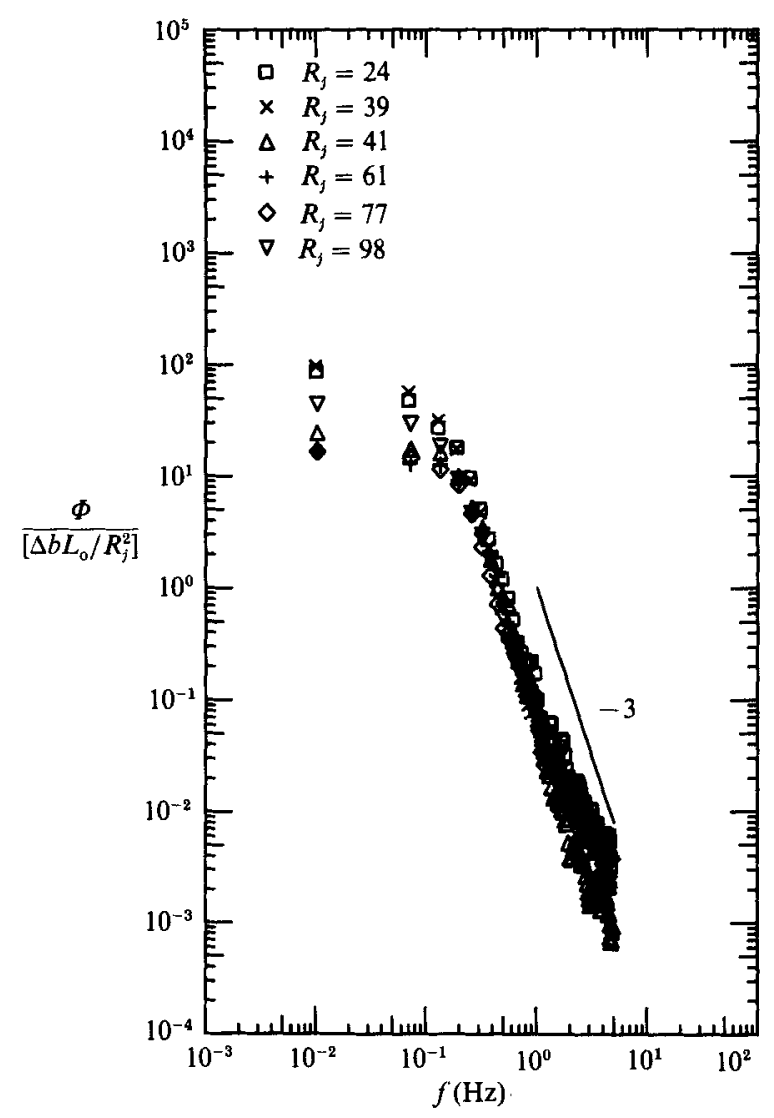

FIGURE 13. Normalized internal wave spectra $\Phi /\left(\Delta b L_{0} / R_{j}^{2}\right)$.

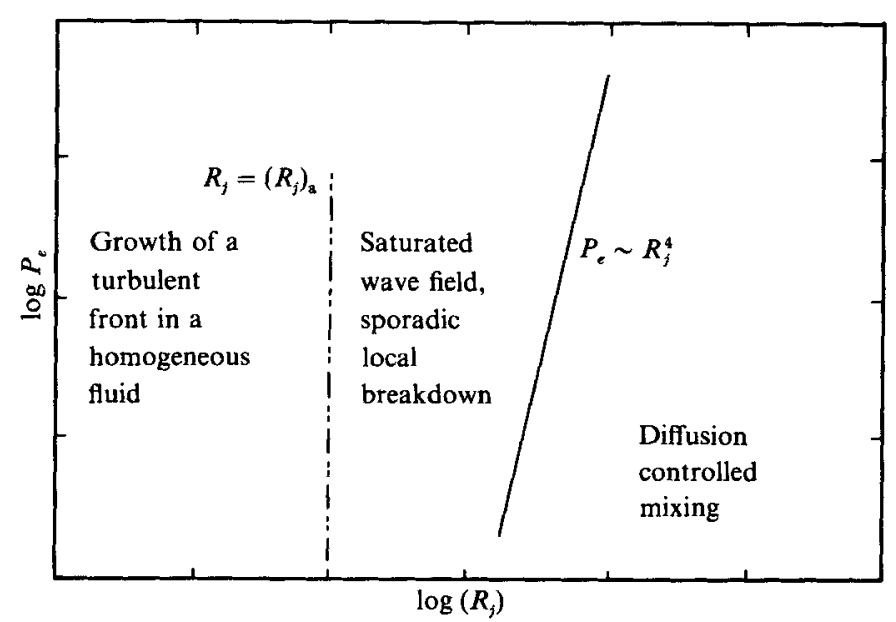

Figure 14. Different entrainment regimes for various values of the Péclet number $P_{e}$ and the overall Richardson number $R_{j}$. 
In order to calculate the rate of entrainment at a shear-free density interface, we have to determine (i) the volume of fluid entrained in individual mixing events and (ii) the frequency of occurrence of these events, $f_{\mathrm{m}}$. The above theory indicates that the lengthscale of the interfacial instabilities is of the same order as the thickness of the interfacial layer. This suggests that the volume of fluid entrained per unit area per event is proportional to $h_{\mathrm{c}}$. It was not possible to determine $f_{\mathrm{m}}$ in the experiments, but in general, it may be expressed as $f_{\mathrm{m}} \tau_{\mathrm{o}}=g\left(R_{j}\right)$ where $g$ is some function. Furthermore, we may write:

$$
\begin{gathered}
\left.u_{\mathrm{e}} \sim \text { (volume entrained per event per unit area) }\right) \times(\text { frequency of occurrence of } \\
\text { mixing events })
\end{gathered}
$$

or

$$
u_{\mathrm{e}} \sim\left(h_{\mathrm{c}}\right) f_{\mathrm{m}}
$$

thus,

$$
u_{\mathrm{e}} /\left[\sigma_{u}\right]_{\mathrm{o}} \sim h_{\mathrm{c}} f_{\mathrm{m}} /\left[\sigma_{u}\right]_{\mathrm{o}} \sim f_{\mathrm{m}} \tau_{\mathrm{o}} R_{j}^{-2} .
$$

The experiments of Fernando \& Long (1983) and Folse et al. (1981) indicate that $u_{\mathrm{e}} /\left(\sigma_{u}\right)_{\mathrm{o}} \sim R_{j}^{-\frac{7}{4}}$ whereas Hopfinger \& Toly (1976), Turner (1968) and this study found that $u_{\mathrm{e}} /\left(\sigma_{u}\right)_{0} \sim R_{j}^{-\frac{\mathrm{s}}{2}}$. These results imply either that $f_{\mathrm{m}} \tau_{\mathrm{o}} \sim R_{j}^{-\frac{1}{4}}$ or $f_{\mathrm{m}} \tau_{\mathrm{o}} \sim R_{j}^{-\frac{1}{2}}$ respectively. We may thus conclude that the frequency of mixing events is a decreasing function of $R_{j}$, a result which is in qualitative agreement with our visual observations in figure 1 .

It is anticipated that the decrease of $h_{\mathrm{c}} / L_{\mathrm{o}}$ with $R_{j}$ cannot continue indefinitely. We suggest that a lower limit on $h_{\mathrm{c}}$ at sufficiently high $R_{j}$ is determined by molecular diffusion. A possible scaling of the interfacial thickness under these conditions is $h_{\mathrm{c}} \sim\left(\kappa \tau_{\mathrm{o}}\right)^{\frac{1}{2}}$ which yields $h_{\mathrm{c}} / L_{\mathrm{o}} \sim P_{e}^{-\frac{1}{2}}$. The latter result was observed in heat stratified experiments when the interface is governed by molecular diffusion (Crapper \& Linden 1974). The above conjecture implies the existence of a cut-off region in which the processes determining the interfacial structure are altered. The cut-off point is obtained when $h_{\mathrm{c}} \sim L_{\mathrm{o}} R_{j}^{-2}$ becomes of the same order as $\left(\kappa \tau_{\mathrm{o}}\right)^{\frac{1}{2}}$, i.e. $L_{\mathrm{o}} R_{j}^{-2} \sim\left\{\kappa L_{\mathrm{o}}\right\}$ $\left.\left[\sigma_{u}\right]_{0}\right\}^{\frac{1}{2}}$. This yields an $R_{j} \sim P_{e}^{\frac{1}{4}}$ criterion for the onset of diffusive effects. In figure 14 we plot $R_{j} P_{e}^{-\frac{1}{4}}=$ constant (roughly estimated to be about 4 ) in the $\left(P_{e}, R_{j}\right)$-plane. Below the line and sufficiently far from it, we expect the interfacial structure to be governed by molecular effects. Sufficiently above the line the interfacial structure can be described by the local wave breakdown model as discussed in this paper. It is also worth noting that for small enough $R_{j}$, say $R_{j}<\left(R_{j}\right)_{\mathrm{a}}$, the buoyancy effects are not important and the upper layer grows in a similar fashion to a turbulent front in a homogeneous fluid. The value of $\left(R_{j}\right)_{\mathrm{a}}$ based on Turner's (1968) measurements is estimated at about 1 .

\section{Discussion}

This paper presents a simple model for turbulent entrainment at a shear-free density interface. The entrainment process and the interfacial structure are proposed to be, in general, functions of Péclet and overall Richardson numbers. The model suggests the existence of three distinct entrainment regimes where the interfacial dynamics are governed by different force balances. At very low $R_{j}$, i.e. $R_{j}<\left(R_{j}\right)_{\mathrm{a}}$, it is expected that the buoyancy effects are negligible and the mixed layer grows in a similar fashion to a turbulent front in a homogeneous fluid. For $\left(R_{j}\right)_{\mathrm{a}} \ll R_{j} \ll \beta P_{e}^{\frac{1}{4}}$, where $\beta$ is a constant, the interface may be characterized by the existence of a saturated wave field and the occurrence of intermittent and sporadic local instabilities that provide a mechanism for fluid mixing. At $R_{j} \gg \beta P_{e}^{\frac{1}{4}}$ the interfacial structure is governed by molecular diffusion. 
Although no experiments were carried out within the last range, our experiments suggest that due to the sharpening of the interface with increasing $R_{j}$, molecular effects are bound to become important at sufficiently high $R_{j}$. For example, a simple calculation shows that for an $h_{\mathrm{c}}$ of about $0.05 \mathrm{~cm}$, as obtained in our experiments at $R_{j}=98, \kappa / h_{\mathrm{e}}$, which presents an estimate of the molecular flux, is about $2 \times 10^{-4} \mathrm{~cm} / \mathrm{s}$. The measured $u_{\mathrm{e}}$ at $R_{j}=98$ is $8 \times 10^{-4} \mathrm{~cm} / \mathrm{s}$. This implies that the molecular flux contributes a significant amount to the total mass flux at $R_{j}=98$. Actually, $h_{\mathrm{c}}=0.05 \mathrm{~cm}$ at $R_{j}=98$ provides a high estimate for the interfacial thickness. This is due to the limited spatial resolution of our equipment as discussed in $\$ 4$. The above calculation suggests that molecular effects become important at $R_{j} \sim 10^{2}$ for $P_{e} \sim 10^{5}$. Turner's (1968) results suggest that molecular effects become important at $R_{i} \sim 10$ for his heat-stratified experiments. $P_{e}$ in those experiments is estimated to be about a factor of 2000 lower than our experiments. The $P_{e} \sim R_{j}^{4}$ scaling for the onset of diffusive effects implies that, based on Turner's findings, we should expect diffusive effects to become significant at $R_{i} \sim 10(2000)^{\frac{1}{4}} \sim 50$ in our experiments. Considering the different methods for obtaining the integral lengthscale of the turbulence in the calculations of $R_{j}$ and $R_{i}$, and taking into account that the point at which the diffusive effects become important is not defined very precisely, the $P_{e} \sim R_{j}^{4}$ relationship seems to provide a good scaling for the onset of molecular effects when heat and salt experiments are compared.

When the interface is governed by molecular diffusion, i.e. sufficiently below the $R_{j} P_{e}^{-\frac{1}{4}}$ line, we anticipate that the rate of entrainment will be proportional to both the concentration gradient and the appropriate diffusivity $\kappa$, i.e. $u_{\mathrm{e}} \sim \kappa / h_{\mathrm{c}}$. Denton \& Wood (1981) had previously used a similar relationship with $h \sim l_{\mathrm{o}}$ (or alternatively $h_{\mathrm{c}} \sim L_{\mathrm{o}}$ ) as determined by Crapper \& Linden (1974) at high $P_{\ell}$. This leads to a $u_{\mathrm{e}} /\left(\sigma_{u}\right)_{\mathrm{o}} \sim P_{e}^{-1}$ relationship. If we use $h_{\mathrm{c}} \sim\left(\kappa \tau_{\mathrm{o}}\right)^{\frac{1}{2}}$, as suggested by our experiments, we obtain $u_{\mathrm{e}} /\left(\sigma_{u}\right)_{0} \sim P_{e}^{-\frac{1}{2}}$, a result which is in agreement with the findings of Fortescue \& Pearson (1967), who determined the rate of entrainment at high $R_{j}$ in the context of gas adsorption into a water surface.

The model we are proposing is similar to the one presented by Denton \& Wood (1981) but with the following major exceptions: (i) our model is valid at all $P_{e}$ as opposed to the low $P_{e}$ limitation in Denton \& Wood's model and (ii) when the interface is governed by molecular diffusion we suggest a $u_{\mathrm{e}} /\left(\sigma_{u}\right)_{0} \sim P_{e}^{-\frac{1}{2}}$ as opposed to the $u_{\mathrm{e}} /\left(\sigma_{u}\right)_{0} \sim P_{e}^{-1}$ relationship proposed by Denton and Wood. The differences between the $P_{e}^{-\frac{1}{2}}$ and $P_{e}^{-1}$ relationships may be significant. In particular, the $P_{e}^{-\frac{1}{2}}$ dependence, when taken in conjunction with $h_{\mathrm{c}} / L_{\mathrm{o}} \sim R_{j}^{-2}$, provides a criterion for the onset of molecular effects that is in agreement with the experimental findings as discussed above. On the other hand, the $u_{e} /\left(\sigma_{u}\right)_{o} \sim P_{e}^{-1}$ relationship, when used with $h / l_{\mathrm{o}} \sim$ constant, cannot predict the prevalence of molecular effects at high $P_{e}$ as observed in our experiments.

Turner (1979) had reported that in heat stratified experiments by C. G. H. Rooth, where $P_{e}$ was varied over a wide range, an $R_{j}^{-\frac{3}{2}}$ range was observed. As $P_{e}$ was reduced, the curves broke away from the $R_{j}^{-\frac{3}{2}}$ line at successively lower values of $R_{j}$, rising above it with decreased slope as molecular diffusion increases the transfer rate. This last observation is in qualitative agreement with our model.

Our model suggests that for $\left(R_{j}\right)_{\mathrm{a}} \ll R_{j} \ll \beta P_{e}^{\frac{1}{4}}$ the turbulence in the upper layer induces the internal wave field at the interface. Furthermore, it is the breakdown of these internal waves that generates local patches of turbulent fluid that are responsible for mixing. This turbulence-wave-turbulence model is different from some earlier ideas about mixing near a shear-free density interface, which postulated that the large eddies in the mixed layer are directly responsible for entraining 
unstirred layer fluid. For instance, Turner (1968) suggested that the mixed layer turbulent eddies directly entrain unstirred layer fluid which becomes ultimately incorporated in the mixed layer. Moreover, Linden (1975) suggested that the rate of change of potential energy is proportional to the kinetic energy flux near the interface. Such an energy argument indicates that the kinetic energy of the eddies is directly converted into potential energy as the eddies impinge on the interface. The work of Linden (1973), in which he studied the effects of vortex rings impinging on a sharp density interface, also suggests that the entrainment occurs after each eddy impinges on the density interface. Although Linden (1973) postulated that the mixing occurs after the recoil of the interface and not by direct entrainment of fluid by the large eddies, the main idea is that mixing occurs after the arrival of every eddy at the interface. However, our flow images in figure 1, the flattening of the eddies near the density interface (Hannoun et al. 1988), and the observations concerning the internal wave field in $\S 4$, all suggest that the mixing is a result of local wave breakdown and not the direct entrainment of unstirred layer fluid by the individual large turbulent eddies. The major idea in our work is that the mixing is occurring sporadically and intermittently and is related to the mixed-layer turbulence only through the internal wave field. Moreover, it is also thought that the turbulence in the upper layer is not very essential for the growth and instability of the internal wave field. Alternatively, irrotational disturbances in one fluid layer may yield similar interfacial structure and dynamics as in our experiment. Actually, Phillips' theory assumes irrotational flow near the density interface. The close agreement between our experimental results and the theory suggests that the rotational aspect of the oscillating grid-induced turbulence may not be essential to the problem.

Although the large eddies of turbulence are flattened at the density interface (Hannoun et al. 1988), and the mixing in our experiments occurs intermittently as postulated by Long (1978), the model proposed in $\$ 5$, is essentially different from Long's arguments. Two major differences between our experimental results and Long's model are in the functional dependence of the interfacial wave velocities and the interfacial thickness on the overall Richardson number. In our experiments, we find that $h_{\mathrm{c}} / L_{\mathrm{o}}$ is a fast decreasing function of $R_{j}$ as opposed to $h / l_{\mathrm{o}}=$ constant in Long's model. Moreover, we find that $w_{\mathrm{i}} /\left[\sigma_{u}\right]_{0} \sim R_{j}^{-\frac{1}{2}}$ as opposed to $w_{\mathrm{h}} /\left(\sigma_{u}\right)_{\mathrm{o}} \sim R_{i}^{-\frac{1}{4}}$ as predicted by Long where $w_{\mathrm{h}}$ is defined as the r.m.s. vertical velocity of the interfacial waves and $w_{\mathrm{i}}$ is the average r.m.s. velocity inferred from the vertical displacement of the concentration contour. Although the definitions of $w_{\mathrm{i}}$ and $w_{\mathrm{h}}$ may be slightly different, the $w_{\mathbf{i}} /\left[\sigma_{u}\right]_{0} \sim R_{j}^{-\frac{1}{2}}$ relationship provides a better fit to our data than a $w_{\mathbf{i}} /\left[\sigma_{u}\right]_{0} \sim R_{j}^{-\frac{1}{4}}$ relationship. Furthermore, Long's model cannot account for the significant molecular mass flux that was observed in our experiments at $P_{e} \sim 10^{5}$ and $R_{j} \sim 10^{2}$.

The realization that molecular effects might be important even at $P_{e} \sim 10^{5}$ provides a possible interpretation of some of the observations of Wolanski \& Brush (1975). They suggested that the rate of entrainment is a function of both an overall Richardson number and a Prandtl number $P_{r}=\nu / \kappa$. An inspection of their entrainment rate versus Richardson number plots suggests that the various curves corresponding to different solutes (except for the case of clay as a 'solute') may be viewed as breaking away from an $R_{j}^{-n}$ line (with $n$ about 2) at values of $R_{j}$ that become successively higher as $P_{r}$ (and consequently $P_{e}$, since $v$ is almost constant) increases. This is in qualitative agreement with our model. A more quantitative comparison is difficult to make because the errors involved in translating Wolanski $\&$ Brush's form of the overall Richardson number to a form similar to the one used 
in this analysis might be large. It is worth mentioning that Wolanski \& Brush concluded that their non-dimensionalized entrainment rates are independent of $P_{e}$. However, our model predicts that the dependence of the entrainment rate on $P_{e}$ is variable and depends on the location in the $\left(P_{e}, R_{j}\right)$-plane. In any event, our model shows that the dependence on $P_{e}$ is slow and in the extreme case is given by $u_{\mathrm{e}} /\left[\sigma_{u}\right]_{\mathrm{o}} \sim P_{e}^{-\frac{1}{2}}$. In suggesting that the entrainment rate is independent of $P_{e}$, Wolanski \& Brush varied $P_{e}$ by about a factor of 3 . If we take into account the amount of scatter in their data points we cannot rule out a $P_{e}^{-m}$ dependence with $m<\frac{1}{2}$.

Finally, it is worth noting that the mixing events were almost always associated with the crest of an apparent wave, consistent with a wave breaking phenomenon. It would be of interest to place the turbulent mixing layer below the quiescent layer and see if this were still the case.

In conclusion, the recent work of Carruthers \& Hunt (1986) is noted. That work describes an analysis of the mixing at the surface of a turbulent layer bounded above by a linearly stratified fluid, a configuration they describe as a class (i) flow and with little similarity to the problem studied here. Our work falls into their category of class (ii) flows, for which a subsequent analysis is promised.

The authors would like to thank Dr D. Papantoniou for setting up the data acquisition system associated with the Reticon camera. We would also like to thank Drs J. Skjelbreia and M. Koochesfahani for their help, Professor J. Imberger for his valuable ideas while spending a sabbatical year at Caltech, and Dr H. J. S. Fernando for his support and friendship. The help of Dr P. Papanicolaou and the entire staff of the W. M. Keck Hydraulics Laboratory is deeply appreciated. The work was supported by US National Science Foundation Grant No. MSM84-12641, A01.

\section{REFERENCES}

Bouvard, M. \& Dumas, H. 1967 The measurement of turbulence in water by the hot wire method. Part 2: Measurement procedure and results. La Houille Blanche 22, 723.

Carruthers, D. J. \& Hunt, J.C. R. 1986 Velocity fluctuations near an interface between a turbulent region and a stably stratified layer. J. Fluid Mech. 165, 475.

Crapper, P. F. \& Linden, P. F. 1974 The structure of turbulent density interfaces. J. Fluid Mech. 65,45 .

Cromwell, T. 1960 Pycnoclines created by mixing in an aquarium tank. J. Mar. Res. $18,73$.

Denton, R. A. \& Wood, I. R. 1981 Penetrative convection at low Péclet number. J. Fluid Mech. 113, 1 .

Dickey, T. D., Hartman, B., Hammond, D. \& Hurst, E. 1984 A laboratory technique for investigating the relationship between gas transfer and fluid turbulence. In Gas Transfer at Water Surfaces (ed. W. Brutsaert \& G. H. Jirka), pp. 93-100. Reidel.

Fernando, H.J.S. 1986 Molecular diffusive effects in stratified turbulent mixing. In

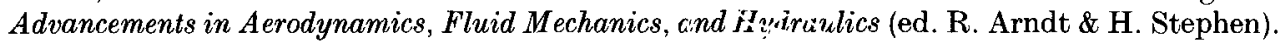
ASCE.

Fernando, H. J. S. \& Long, R. R. 1983 The growth of a grid-generated turbulent mixed layer in a two-fluid system. J. Fluid Mech. 133, 377.

Fernando, H. J. S. \& Long, R. R. 1985 On the nature of the entrainment interface of a two-layer fluid subjected to zero-mean-shear turbulence. J. Fluid Mech. 151, 21.

Fischer, H. B., List, E. J., Koh, R. C. Y., Imberger, J. \& Brooks, N. H. 1979 Mixing in Inland and Coastal Waters. 483 pp. Academic.

Folse, R. F., Cox, T. P. \& Schexnayder, K. R. 1981 Measurements of the growth of a turbulently mixed layer in a linearly stratified fluid. Phys. Fluids 24, 396. 
Fortescue, G. E. \& Pearson, J. R. A. 1967 On gas absorption into a turbulent fluid. Chem. Engng Sci. 22, 1163.

Gartrell, G. 1979 Studies on the mixing in a density-stratified shear flow. Ph.D. thesis. California Institute of Technology.

Hannoun, I. A. 1987 Turbulent mixing in stably-stratified fluids subjected to zero-mean shear. Ph.D. thesis. California Institute of Technology.

Hannoun, I. A., Fernando, H. J. S. \& List, E. J. 1988 Turbulence structure near a sharp density interface. J. Fluid Mech. 189, 189.

Hopfinger, E. J. \& Linden, P. F. 1982 Formation of thermoclines in zero-mean shear turbulence subjected to a stabilizing buoyancy flux. J. Fluid Mech. 114, 157.

Hopfinger, E. J. \& Toly, J. A. 1976 Spatially decaying turbulence and its relation to mixing across density interfaces. J. Fluid Mech. 78, 155.

Hunt, J. C. R. \& Graham, J. M. R. 1978 Free-stream turbulence near plane boundaries. J. Fluid Mech. 84, 209.

Kantha, L. H.\& Long, R. R. 1980 Turbulent mixing with stabilizing buoyancy flux. Phys. Fluids $23,2142$.

Kantha, L. H., Phillips, O. M. \& Azad, R. S. 1977 On turbulent entrainment at a stable density interface. J. Fluid Mech. 79, 753.

Kato, H. \& Phillifs, O. M. 1969 On the penetration of a turbulent layer into a stratified fluid. J. Fluid Mech. 37, 643.

Koochesfahani, M. M. 1984 Experiments on turbulent mixing and chemical reaction in a liquid mixing layer. Ph.D. thesis. California Institute of Technology.

Linden, P. F. 1973 The interaction of a vortex ring with a sharp density interface: a model for turbulent entrainment. J. Fluid Mech. 60, 467.

Linden, P. F. 1975 The deepening of a mixed layer in a stratified fluid. J. Fluid Mech. 71, 385.

LiU, H. T., Lin, J.-T., Delisi, D. P.\& Robren, F. A. 1977 Application of a fluorescence technique to dye-concentration measurements in a turbulent jet. NBS Special Publication 484 in Proc. Symp. on Flow in Open Channels and Closed Conduits held at NBS, Gaithersburg, MD, February 1977.

Long, R. R. 1978 A theory of mixing in stably stratified fluids. J. Fluid Mech. 84, 113.

McDovgall, T. J. 1979 Measurements of turbulence in a zero-mean shear mixed layer. J. Fluid Mech. 94, 409.

Miles, J. W. 1961 On the stability of heterogeneous shear flows. J. Fluid Mech. 10, 496.

Papanicolaou, P. N. 1984 Mass and momentum transport in a turbulent buoyant vertical axisymmetric jet. PhD thesis. California Institute of Technology.

Papantoniou, D. P. 1986 Observations in turbulent buoyant jets by use of laser-induced fluorescence. $\mathrm{PhD}$ thesis. California Institute of Technology.

Phillips, O. M. 1977 Dynamics of the Upper Ocean. Cambridge University Press.

Reticon 1981 LC300A Camera Manual EG \& G Reticon.

Rouse, H. \& Dodu, J. 1955 Turbulent diffusion across a density discontinuity. La Houille Blanche 4, 522 .

Scavia, D. \& Robertson, A. (ed.) 1981 Perspectives on Lake Ecosystem Modeling. Ann Arbor Science.

Thompson, S. M. \& Turner, J. S. 1975 Mixing at an interface due to turbulence generated by an oscillating grid. J. Fluid Mech. 67, 349.

Turner, J.S. 1968 The influence of molecular diffusivity on turbulent entrainment across a density interface. J. Fluid Mech. 33, 639.

TURner, J.S. 1979 Buoyancy Effects in Fluids. Cambridge University Press.

Weast, R. C. (ed.) 1976 Handbook of Chemistry and Physics. 56th Edition. C.R.C. Press.

Wolanski, E. J. \& BRUSh, L. M. 1975 Turbulent entrainment across stable density step structures. Tellus 27, 259.

Wu, J. 1973 Wind induced turbulent entrainment across a stable density interface. J. Fluid Mech. $61,275$. 